\title{
A coupled FEM/DEM approach for hierarchical multiscale modelling of granular media
}

\author{
Ning Guo and Jidong Zhao*,† \\ Department of Civil and Environmental Engineering, The Hong Kong University of Science and Technology, \\ Clearwater Bay, Kowloon, Hong Kong
}

\begin{abstract}
SUMMARY
A hierarchical multiscale framework is proposed to model the mechanical behaviour of granular media. The framework employs a rigorous hierarchical coupling between the FEM and the discrete element method (DEM). To solve a BVP, the FEM is used to discretise the macroscopic geometric domain into an FEM mesh. A DEM assembly with memory of its loading history is embedded at each Gauss integration point of the mesh to serve as the representative volume element (RVE). The DEM assembly receives the global deformation at its Gauss point from the FEM as input boundary conditions and is solved to derive the required constitutive relation at the specific material point to advance the FEM computation. The DEM computation employs simple physically based contact laws in conjunction with Coulomb's friction for interparticle contacts to capture the loading-history dependence and highly nonlinear dissipative response of a granular material. The hierarchical scheme helps to avoid the phenomenological assumptions on constitutive relation in conventional continuum modelling and retains the computational efficiency of FEM in solving large-scale BVPs. The hierarchical structure also makes it ideal for distributed parallel computing to fully unleash its predictive power. Importantly, the framework offers rich information on the particle level with direct link to the macroscopic material response, which helps to shed lights on cross-scale understanding of granular media. The developed framework is first benchmarked by a simulation of single-element drained test and is then applied to the predictions of strain localisation for sand subject to monotonic biaxial compression, as well as the liquefaction and cyclic mobility of sand in cyclic simple shear tests. It is demonstrated that the proposed method may reproduce interesting experimental observations that are otherwise difficult to be captured by conventional FEM or pure DEM simulations, such as the inception of shear band under smooth symmetric boundary conditions, non-coaxial granular response, large dilation and rotation at the edges of shear band and critical state reached within the shear band. Copyright (C) 2014 John Wiley \& Sons, Ltd.
\end{abstract}

Received 14 April 2014; Accepted 25 April 2014

KEY WORDS: hierarchical multiscale method; coupled FEM/DEM model; granular media; strain localisation; non-coaxiality; fabric anisotropy

\section{INTRODUCTION}

Granular materials underpin the operation and performance of key infrastructures in civil and mining engineering and energy extraction industries. Under confined or unconfined shear, a granular material may exhibit complicated macroscopic behaviours that are difficult to characterise, such as state dependency, strain localisation, strength anisotropy, non-coaxiality, solid-flow phase transition (e.g. liquefaction) and critical state. These macroscopic behaviours reflect nontrivial complex microstructural mechanisms at the particle level of the material. A granular material has conventionally been treated within the framework of continuum mechanics where the discrete nature of the material is smeared out and the material body is treated as a homogenised continuum, based on which

\footnotetext{
*Correspondence to: Jidong Zhao, Department of Civil and Environmental Engineering, The Hong Kong University of Science and Technology, Clearwater Bay, Kowloon, Hong Kong.

†E-mail: jzhao@ust.hk
} 
various continuum constitutive models have been developed and have gained certain success in capturing the behaviour of granular media. Recent progresses in continuum modelling of granular materials enable us to capture features such as fabric anisotropy and fabric evolution $[1,2]$, noncoaxiality [3-6] and strain localisation [7]. The implementation of these continuum models into numerical tools such as FEM renders them truly predictive in solving practical BVPs. Continuum constitutive models hence remain the most effective and commonly used approaches for engineering applications so far. One major criticism for most continuum models, however, is that their constitutive relations may contain various model parameters that are phenomenological in nature without clear physical meanings and/or difficult to calibrate. Inadequate consideration of relevant information from the grain scale of a granular material may be an attributable reason. Meanwhile, discrete element method (DEM) [8] has become a popular tool in the study on granular media recently. DEM respects the discrete nature of a granular material by tracing the kinetics and kinematics of the constituent particles based on simple, physically intuitive and experimentally easily verifiable contact and friction laws (e.g. linear or Hertz contact law and Coulomb's friction) to describe the interparticle contacts. Based on homogenisation [9-13] over an assembly of discrete particles, DEM is able to reasonably reproduce many of the typical mechanical responses of a granular material and to provide rich information at the microscopic level of the material. To faithfully reproduce the typical behaviour of granular media, however, DEM needs to simulate an assembly involving extremely large number of granular particles, which may incur exceedingly great computational cost. For example, to realistically simulate a typical laboratory test on sand (e.g. conventional triaxial test), several to over 10 million of sand particles have to be generated to form a DEM sample, which is simply beyond the computing capacity that can be routinely accessed. In view of its difficulty to replicate an element test, it is quickly realised that it is currently unrealistic for DEM to solve a practical problem of engineering scale. It is hence desirable to develop an effective method to fully utilise the advantages of both the continuum approaches and DEM and meanwhile to avoid or mitigate their respective pitfalls. Multiscale modelling approach provides a viable solution to this issue.

There have been considerable progresses in combining a continuum-based method and a discontinuum-based one for material modelling, particularly in material science where various homogenisation techniques have been successfully developed to link different length scales of a material for integrated characterisation of material behaviour. Many of these studies have been targeted at designing engineered or new materials with identifiable microstructure to achieve optimal performance for various purposes. Granular materials differ considerably from these materials in at least two aspects. First, a periodic microstructure is generally not available for a granular material due to its randomness at large. Second, the behaviour of granular media is state dependent and loading-path specific. It is hence difficult to find a once-for-all microstructure from which the macroscopic properties can be derived via the homogenisation methods used in material science. These issues can be effectively tackled by the hierarchical multiscale computational approach. Specifically, a computational hierarchy can be constructed such that a BVP can be solved by FEM on the macroscale, whereas the constitutive relation is derived from DEM modelling at each integration point of the FEM [14-16]. Notably, the strategy is similar to the two-scale modelling approach by [17-19] on the simulation of highly heterogeneous materials (e.g. with microstructures such as random pores and inclusions) wherein both scales employed FEM (called $\mathrm{FE}^{2}$ ). For granular media, this hierarchical multiscale modelling strategy may help to avoid assuming any phenomenological constitutive relation for the continuum modelling by FEM and to maintain its predictive capability. It meanwhile overcomes the limit on simulating scale of DEM. Indeed, the hierarchical modelling concept, once successfully materialised to a predictive model, may effectively fill the long-standing gap between the continuum constitutive modelling and micromechanics-based discrete modelling and helps to enrich the general arsenal for granular material modelling.

There have been limited attempts on this topic. Meier and coworkers [14, 20], for example, have implemented a coupled FEM/DEM scheme with two demonstrative examples. Notably, however, they adopted a Taylor assumption at the microscale by restricting the displacements of the particles with a prescribed deformation, that is, only affine displacements are allowed for the particles. This continuum-based assumption may cause unrealistically stiffer and less dissipative responses 
for a granular medium. Andrade and coworkers [15, 21, 22] more recently developed an interesting bridging scheme to simulate strain localisation problems in granular media. Their approach retained a simple plasticity model at the macroscale with two constitutive parameters (friction angle and dilation angle) and tracked their evolutions based on data either from a DEM model or a concurrently performed laboratory test. Whilst offering rather encouraging results, their approach can be regarded as a partial bypassing of the phenomenological nature due to the retaining of plasticity parameters. Some more recent progresses have been made towards developing a fully coupled hierarchical model aiming at totally bypassing the phenomenological assumptions in continuum constitutive models $[16,23,24]$. These studies, however, remain rather tentative and experimental in nature and need substantial improvements in both computational algorithms and detailed aspects on calibrations and validations. In this study, we aim to develop the aforementioned hierarchical concept into a predictive multiscale modelling framework to model the behaviour of granular media and relevant engineering problems. A hierarchical multiscale approach based on rigorous coupling between the FEM and the DEM will be formulated by highlighting the computational innovations. The predictive capability of the proposed framework will be showcased by solving BVPs in geomechanics under both monotonic and cyclic loadings. Its advantage in bridging multiple scales will be demonstrated by correlation of the macroscopic observations in these examples and their underlying microstructural mechanisms. With an integration of general and robust numerical techniques and solution procedures, the obtained hierarchical multiscale framework is indeed a general purpose one so that it may be potentially applied to broad fields dealing with granular materials other than geomechanics. Note that the proposed hierarchical approach may be regarded as an inversion of and complementary to the so-called combined finite-DEM (FDEM) pioneered by Munjiza and coworkers [25, 26] and demonstrated elsewhere [27, 28]. In FDEM, discrete elements representing individual granular particles or rock blocks are meshed into finite elements and are subjected to deformation and potential fragmentation due to static contact or dynamic collision. Meanwhile, the hierarchical strategy differs essentially from the concurrent multiscale method [29-32], which are widely discussed in the literature. In a concurrent multiscale approach, the problem domain is usually decomposed into a larger continuum subdomain, which undergoes relatively small deformations and a smaller discrete subdomain where potential fracturing, cracking or other discontinuous behaviour may be of interest.

The paper is organised as follows. We first present the solution procedure and formulations of the hierarchical multiscale computational framework and then benchmark it using a single element test (SET). The predictive capacity of the developed tool is then examined by simulation of a monotonic biaxial compression test on sand, with particular attention being placed on the analyses of the initiation and development of strain localisation in the tested specimen. We also showcase the performance of the multiscale approach in simulating cyclic simple shear test on sand. We then conclude the paper with further discussion. For the notations and symbols, blackboard bold letters denote rank four tensors and boldface letters for matrices, rank two tensors and vectors. ' $:$ ' denotes the double contraction between two tensors, and ' $\otimes$ ' denotes the dyadic product between two vectors. ' $\nabla$ ' and ' $\nabla$ '' are used for gradient and divergence notations, respectively. 'tr' and 'dev' take the trace and the deviator of a tensor, respectively.

\section{FORMULATION AND SOLUTION PROCEDURE}

\subsection{Finite element formulation}

In this coupled FEM/DEM framework on hierarchical multiscale modelling of granular media, the geometric domain $\Omega$ of a given BVP is first discretised into a suitable FEM mesh. For a quasi-static problem in the absence of body force, the governing equation can be written in the following weak variational form

$$
\int_{\Omega} \sigma: \nabla \boldsymbol{w} \mathrm{d} V=\int_{\partial \Omega_{t}} \boldsymbol{t} \cdot \boldsymbol{w} \mathrm{d} A
$$


where $\sigma$ is the internal stress, $t$ is the boundary traction applied on the domain surface $\partial \Omega_{t}$ and $\boldsymbol{w}$ is the test function that can be chosen as the variation of the primary unknown (i.e. displacement) via the principle of virtual work. After the finite element discretisation, one ends up with the following equation system to be solved,

$$
K \boldsymbol{u}=\boldsymbol{f}
$$

where $\boldsymbol{K}$ is the stiffness matrix, $\boldsymbol{u}$ is the unknown displacement vector at the FEM nodes and $\boldsymbol{f}$ is the nodal force vector lumped from the applied boundary traction. For a typical linear elastic problem, $\boldsymbol{K}$ can be formulated from the elastic modulus, and Equation (2) can be solved directly. Whilst in the case involving nonlinearity such as for granular media where $\boldsymbol{K}$ depends on state parameters and loading history, Newton-Raphson iterative method needs to be adopted and the stiffness matrix is replaced with the tangent matrix $\boldsymbol{K}_{t}$, which is assembled from the tangent operator

$$
\boldsymbol{K}_{t}=\int_{\Omega} \boldsymbol{B}^{T} \boldsymbol{D} \boldsymbol{B} \mathrm{d} V
$$

where $\boldsymbol{B}$ is the deformation matrix (i.e. gradient of the shape function), and $\boldsymbol{D}$ is the matrix form of the rank four tangent operator tensor $\mathbb{D}$. During each Newton-Raphson iteration, both $\boldsymbol{K}_{t}$ and $\boldsymbol{\sigma}$ are updated, and the scheme tries to minimise the residual force $\boldsymbol{R}$ to find a converged solution

$$
\boldsymbol{R}=\int_{\Omega} \boldsymbol{B}^{T} \sigma \mathrm{d} V-\boldsymbol{f}
$$

Clearly, the tangent operator and the stress tensor at each local Gauss integration point are pivotal variables in the aforementioned calculation and need to be evaluated before each iteration and loading step. A continuum-based conventional FEM usually assumes a constitutive relation for the material and derives the tangent matrix and the stress increment based on this constitutive assumption [e.g. using the elasto-plastic modulus $\boldsymbol{D}^{e p}$ in Equation (3) to assemble $\boldsymbol{K}_{t}$ and to integrate stress]. The coupled FEM/DEM multiscale approach obtains the two quantities from the embedded discrete element assembly at each Gauss point and avoids the needs for phenomenological assumptions.

\subsection{Discrete element homogenisation}

A DEM assembly is attached to each Gauss point of the FEM mesh, which serves as the representative volume element (RVE) at the specific point to capture the local material response. The boundary condition (deformation) for each DEM packing is interpolated from the FEM solution (displacement). Then the stress and tangent operators are derived and upscaled to the FEM solver to update the solution. We follow Love's formula in deriving the homogenised Cauchy stress from a discrete element assembly [33]

$$
\sigma=\frac{1}{V} \sum_{N_{c}} \boldsymbol{d}^{c} \otimes \boldsymbol{f}^{c}
$$

where $V$ is the total volume of the assembly, $N_{c}$ is the number of contacts within the volume and $f^{c}$ and $\boldsymbol{d}^{c}$ are the contact force vector and the branch vector connecting the centres of the two contacted particles, respectively (see Figure 1). In defining the direction of branch vector in Figure 1, compression is taken as positive. Although the proposed method is generally three dimensional (3D), for demonstrative purposes, the simulations in the following sections will be made in 2D where both the stress and strain components in the out-of-plane direction are assumed to be zero. Based on Equation (5), two commonly referred quantities - the mean effective stress $p$ and the deviatoric stress $q$, are calculated as follows for the considered two-dimensional (2D) case: 

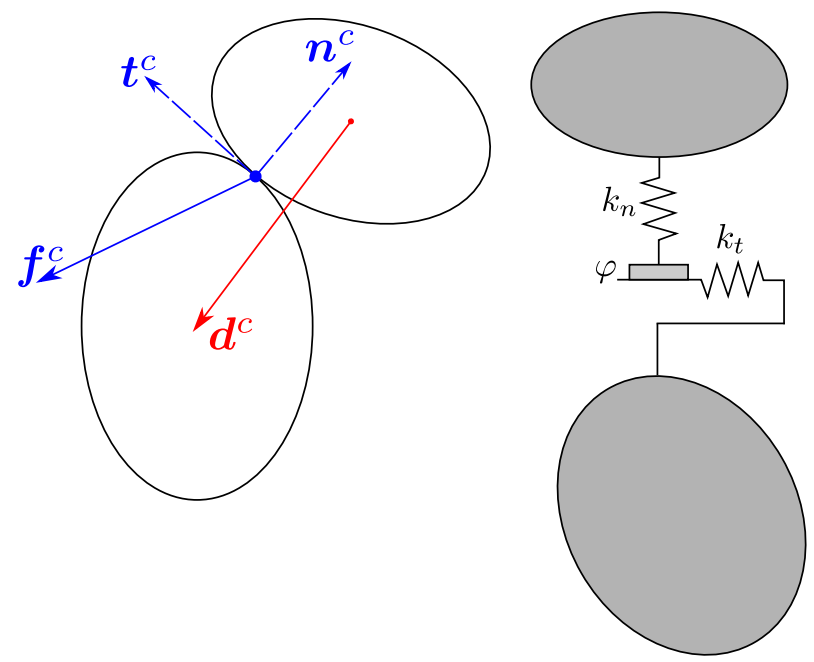

Figure 1. The illustration of a contact in DEM model.

$$
\begin{gathered}
p=\frac{1}{2} \operatorname{tr} \sigma \\
q=\sqrt{\frac{1}{2} s: s}
\end{gathered}
$$

where $s$ is the deviatoric stress tensor, $s=\operatorname{dev} \sigma=\sigma-p \boldsymbol{\delta}$, with $\delta$ being the Kronecker delta.

In deriving the tangent operator, the performances of two different methods are compared. The first one is based on the perturbation method [16]. By adding a small perturbation to the current strain level $\varepsilon^{0}$ and obtaining the stress variation, the perturbation method leads to an approximation of the tangent operator as follows:

$$
\mathbb{D}^{p}=\left.\frac{\partial \boldsymbol{\sigma}}{\partial \boldsymbol{\varepsilon}}\right|_{\boldsymbol{\varepsilon}=\boldsymbol{\varepsilon}^{0}} \cong \frac{\sigma\left(\varepsilon^{0}+\xi \Delta^{k l}\right)-\sigma\left(\varepsilon^{0}\right)}{\xi}
$$

where $\xi$ denotes the magnitude of the perturbation, and $\Delta^{k l}$ is a rank two tensor with all components being zero except the one with superscript $k l$ (the perturbation direction), which is equal to 1 . In the $2 \mathrm{D}$ case, a total of four perturbations are needed to obtain the full tangent operator.

In the second method, we estimate the tangent operator from the homogenised bulk elastic modulus of the DEM assembly. The analytical form based on the assumption of uniform strain field (termed as Taylor assumption by Meier et al. [14]) is given [34-37]:

$$
\mathbb{D}^{e}=\frac{1}{V} \sum_{N_{c}}\left(k_{n} \boldsymbol{n}^{c} \otimes \boldsymbol{d}^{c} \otimes \boldsymbol{n}^{c} \otimes \boldsymbol{d}^{c}+k_{t} \boldsymbol{t}^{c} \otimes \boldsymbol{d}^{c} \otimes \boldsymbol{t}^{c} \otimes \boldsymbol{d}^{c}\right)
$$

where $k_{n}$ and $k_{t}$ are the equivalent normal and tangential stiffnesses describing the contact law of the particles and $\boldsymbol{n}^{c}$ and $\boldsymbol{t}^{c}$ are the unit vectors in the outward normal and tangential directions of a contact, respectively (see Figure 1). Note that the expression applies to both spherical and aspherical particles, as well as to nonlinear contact laws when $k_{n}$ and $k_{t}$ are appropriately defined, that is, $k_{n}:=d f_{n}^{c} / d u_{n}^{c}$ and $k_{t}:=d f_{t}^{c} / d u_{t}^{c}$, where $f_{n}^{c}$ and $f_{t}^{c}$ are normal and tangential components of the contact force, and $u_{n}^{c}$ and $u_{t}^{c}$ are relative normal and tangential displacements of the two particles at a contact. The relative normal displacement $u_{n}^{c}$ is commonly referred to as the overlapping of the two particles. Taking the popular Hertz-Mindlin contact model, for example, the stiffnesses can be 


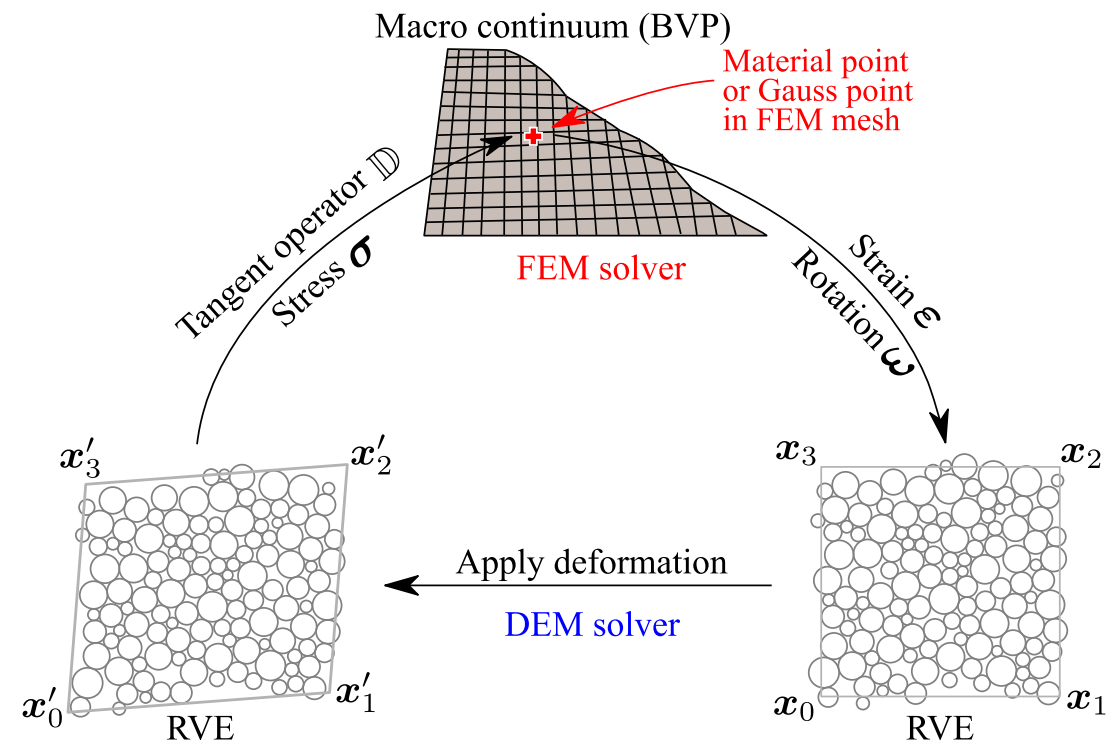

Figure 2. The solution procedure of hierarchical multiscale modelling.

derived as follows: $k_{n}=\frac{G}{1-v} \sqrt{2 r^{*} u_{n}^{c}}$ and $k_{t}=\frac{2 G}{2-v} \sqrt{2 r^{*} u_{n}^{c}}$, where $G$ and $v$ are the shear modulus and Poisson's ratio of the particle material (e.g. quartz), respectively, and $r^{*}$ is the common radius of the contacted particles (i.e. $r^{*}=\frac{2 r_{1} r_{2}}{r_{1}+r_{2}}$ where $r_{1}$ and $r_{2}$ are the radii of the two contacted particles).

\subsection{Multiscale solution procedure}

The hierarchical multiscale modelling procedure is schematically illustrated in Figure 2 and is summarised as the following steps:

(1) Discretise the problem domain by suitable FEM mesh and attach each Gauss point with a DEM assembly prepared with suitable initial state.

(2) Apply one global loading step, that is, imposed by FEM boundary condition on $\partial \Omega$.

(a) Determine the current tangent operator using either Equation (8) or (9) for each RVE.

(b) Assemble the global tangent matrix using Equation (3) and obtain a trial solution of displacement $\boldsymbol{u}$ by solving Equation (2) with FEM.

(c) Interpolate the deformation $\nabla \boldsymbol{u}$ at each Gauss point of the FEM mesh and run the DEM simulation for the corresponding RVE using $\nabla \boldsymbol{u}$ as the DEM boundary conditions.

(d) Derive the updated total stress from Equation (5) for each RVE and use them to evaluate the residual by Equation (4) for the FEM domain.

(e) Repeat the aforementioned steps from (a) to (d) until convergence is reached and finish the current loading step.

(3) March on the next loading step and repeat Step 2.

In interpolating the deformation $\nabla \boldsymbol{u}$ from the FEM solution for DEM boundary conditions in Step 2(c), we consider both the infinitesimal strain $\boldsymbol{\varepsilon}$ and rotation $\boldsymbol{\omega}$

$$
\nabla \boldsymbol{u}=\underbrace{\frac{1}{2}\left(\nabla \boldsymbol{u}+\nabla \boldsymbol{u}^{T}\right)}_{\boldsymbol{\varepsilon}}+\underbrace{\frac{1}{2}\left(\nabla \boldsymbol{u}-\nabla \boldsymbol{u}^{T}\right)}_{\omega}
$$


The corresponding RVE packing will deform according to this prescribed boundary condition. The boundary of any RVE can be described by its cell nodes (four nodes in 2D case, see Figure 2). Suppose the coordinates of one of the nodes before and after deformation are denoted as $\boldsymbol{x}$ and $\boldsymbol{x}^{\prime}$, respectively. The updated coordinates $\boldsymbol{x}^{\prime}$ can be determined from the deformation via

$$
\frac{\partial \boldsymbol{x}^{\prime}}{\partial \boldsymbol{x}}=\nabla \boldsymbol{u}+\boldsymbol{\delta}
$$

For the strain measure used to present the results in the study, the infinitesimal strain $\varepsilon$ is obtained from the symmetric part of the boundary displacement gradient $\nabla \boldsymbol{u}$ [see Equation (10)], based on which the volumetric strain $\varepsilon_{\mathrm{v}}$ and the deviatoric strain $\varepsilon_{q}$ can be derived (for 2D case)

$$
\begin{gathered}
\varepsilon_{\mathrm{v}}=\operatorname{tr} \boldsymbol{\varepsilon} \\
\varepsilon_{q}=\sqrt{2 \boldsymbol{e}: \boldsymbol{e}}
\end{gathered}
$$

where $\boldsymbol{e}$ is the deviatoric strain tensor $\boldsymbol{e}=\operatorname{dev} \boldsymbol{\varepsilon}=\boldsymbol{\varepsilon}-\frac{1}{2} \varepsilon_{\mathrm{v}} \boldsymbol{\delta}$.

It is also instructive to add a few remarks on the evolution of stress from the RVE in Step 2(d). In traditional FEM, the stress is updated based on an incremental manner to tackle the nonlinear material response. If small strain is assumed, the incremental stress-strain relation may potentially cause inaccurate numerical results when large deformation occurs in the material, which calls for an alternative formulation for large deformation. This issue indeed can be naturally circumvented in the current hierarchical framework. In our framework, the DEM assembly at each Gauss point will memorise its past state history (e.g. pressure level, void ratio and fabric structure) and will be solved with the current applied boundary condition (including both stretch and rotation) at each loading and iteration step. Towards the end of each loading step, instead of using an incremental stress update scheme, the total true stress (Cauchy stress) is derived directly over the solved DEM assembly through homogenisation by Equation (5) and is then returned to the FEM solver for the global solution. In this way, we do not have to resort to the use of other objective stress measures to deal with large deformation problems. However, we note that a proper strain measurement is still required and the FEM mesh should not be severely distorted, otherwise, remeshing of the FEM domain will be required (see Appendix for more discussion).

\section{BENCHMARK, CALIBRATION AND PARALLELISATION}

We have implemented the aforementioned hierarchical multiscale approach by coupling two opensource codes-Escript [38] and YADE [39]-for the FEM and DEM computations, respectively. The FEM part follows the conventional displacement driven formulation, which has been briefly summarised in Section 2.1. In the following, the DEM model and the relevant formulations and parameters are first introduced. We will then use a SET under drained biaxial compression to benchmark the proposed approach. The two methods calculating the tangent operator are compared in terms of their performance and efficiency. In this section, We will also introduce the parallelisation of the proposed multiscale framework to fully enhance its predictive capacity.

\subsection{DEM model}

For simplicity, YADE is modified for 2D analyses (e.g. by restraining all degrees of freedom and assuming zero stress components in the out-of-plane direction). Polydisperse particles (cylindrical rods) with radii ranging from 3 to $7 \mathrm{~mm}\left(r_{\text {mean }}=5 \mathrm{~mm}\right)$ are used for the DEM assembly. The thickness of all particles is set to be $100 \mathrm{~mm}$ (10 times of the mean particle diameter). Note that the adopted particle size is scaled-up about 1-2 orders in the DEM simulation as compared with the grain size in real sand, for example, Toyoura sand. A simple linear force-displacement contact 
law in conjunction with Coulomb's friction criterion is employed to describe the stick-slip interparticle contact. Three input parameters are required: Young's modulus $E_{c}$, Poisson's ratio $v_{c}$ and interparticle friction angle $\varphi$, through which the normal and tangential stiffnesses are determined

$$
\begin{gathered}
k_{n}=E_{c} r^{*} \\
k_{t}=v_{c} k_{n}
\end{gathered}
$$

Notably, by assuming a linear contact law, the stiffnesses $k_{n}$ and $k_{t}$ can be directly applied in Equation (9) to calculate $\mathbb{D}^{e}$. The normal and tangential (frictional) forces can then be determined as follows:

$$
\begin{gathered}
\boldsymbol{f}_{n}^{c}= \begin{cases}\mathbf{0}, & \text { if } u_{n}^{c} \leqslant 0 \\
-k_{n} u_{n}^{c} \boldsymbol{n}^{c}, & \text { otherwise }\end{cases} \\
\boldsymbol{f}_{t}^{c}= \begin{cases}-k_{t} \boldsymbol{u}_{t}^{c}, & \text { if } f_{t}^{c} \leqslant f_{n}^{c} \tan \varphi \\
-f_{n}^{c} \tan \varphi \boldsymbol{u}_{t}^{c} / u_{t}^{c}, & \text { otherwise }\end{cases}
\end{gathered}
$$

where the interparticle friction angle $\varphi$ imposes a threshold for the tangential force, and $\boldsymbol{u}_{t}^{c} / u_{t}^{c}$ returns the direction of $\boldsymbol{u}_{t}^{c}$. To ensure quasi-static condition, a simple local non-viscous damping force [40] is added to dissipate kinetic energy

$$
\boldsymbol{f}^{\text {damp }}=-\alpha f^{\text {resid }} \boldsymbol{v} / v
$$

where $\alpha$ is the damping ratio, $\boldsymbol{v}$ is the velocity of the particle, $\boldsymbol{v} / v$ gives the direction of $\boldsymbol{v}$ and $f^{\text {resid }}$ is the residual force accumulated from all the contact forces on the particle. The kinematics of the particles follows the Newton's law of motion and the positions of particles are updated using a time-marching scheme, which requires a critical time step to ensure numerical stability for DEM computations estimated by [41]

$$
\Delta t_{c r}=\sqrt{m_{\min } / k_{n}}
$$

where $m_{\min }$ is the mass of the smallest particle in the assembly. For safety reasons, the time step is set $\Delta t<0.5 \Delta t_{c r}$ in the study. The strain rate for each RVE $\dot{\gamma}=\sqrt{\dot{\boldsymbol{\varepsilon}}: \dot{\boldsymbol{\varepsilon}}}$ is determined from the inertia number [42]

$$
I=2 \dot{\gamma} r_{\text {mean }} \sqrt{\rho_{p} / p}
$$

where $\rho_{p}$ is the particle density. To guarantee quasi-static condition, this number is kept as $I<$ $10^{-3}$. Due to different natures of DEM and FEM calculations, one typical loading step of FEM computation is usually accompanied with hundreds to thousands of DEM computational steps in the multiscale model.

\subsection{Calibration of RVE size}

To determine the size of the RVE (particle number) attached to each Gauss point, a general guideline is that the RVE should be large enough to be representative and to imprint the characteristic microstructure of the material and be meanwhile small enough to ensure computational efficiency and to avoid localisation occurring within the RVE during the loading course. To examine the suitable RVE size for the DEM assembly in our multiscale framework, we follow a similar approach as proposed by Meier et al. [20], by generating RVEs of different sizes but with the same particle 
RVE 100 particles

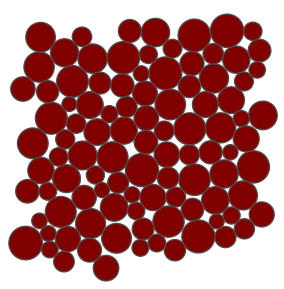

RVE 200 particles

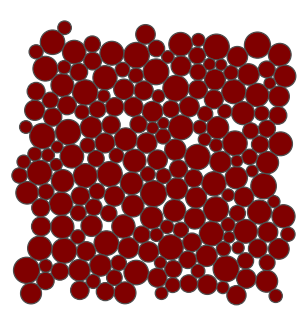

RVE 400 particles

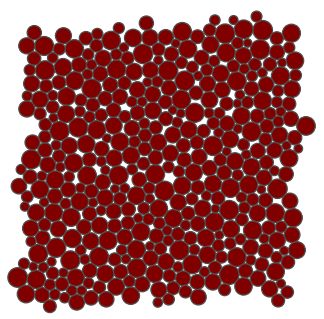

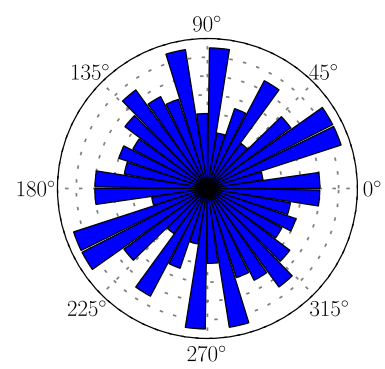
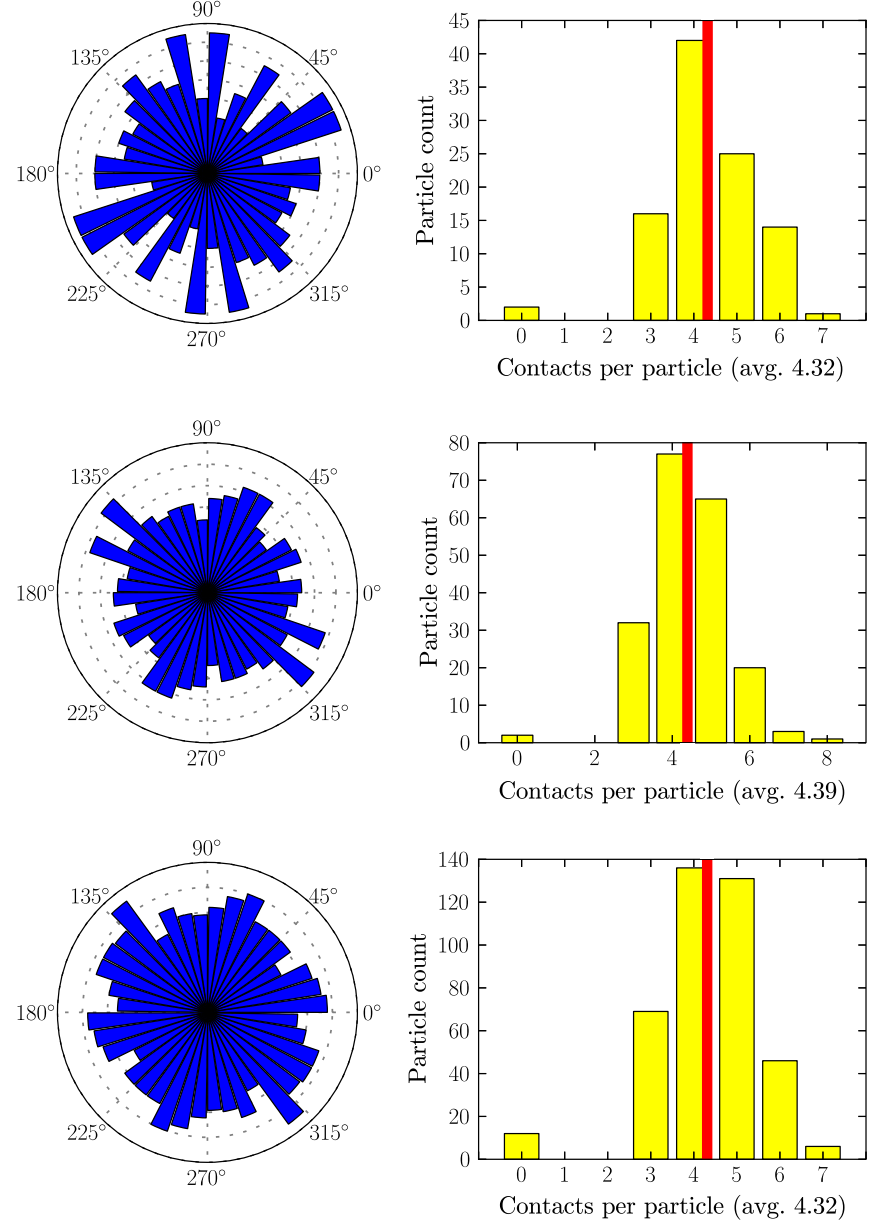

Figure 3. Comparison of different sizes of RVEs.

size distribution to examine the contact normal distribution (termed as fabric, see Section 4.2) of these RVEs under isotropic compression. We compare in Figure 3 the results of three different sizes of RVEs, containing 100, 200 and 400 particles, respectively, under an isotropic pressure of $p_{0}=100 \mathrm{kPa}$. The distributions of contact number per particle in the three RVEs are found rather close, with an average value (termed as coordination number) at around 4.3 for all cases. It is evident, however, that the orientation distribution of the contact normals is more uniform for RVE with more particles. The third RVE containing 400 particles shows a largely isotropic fabric with its rose diagram resembling a near circular shape. This is expected for an isotropic granular material under isotropic compression. In contrast, the other two RVEs show apparent anisotropic fabrics. We hereby favour the third RVE and will use it in the following studies. Relevant parameters of the particles, summarised in Table I, have been so calibrated that the overall behaviour of the RVE is in qualitative $^{\ddagger}$ agreement with laboratory tests on sand. The particle size distribution and a snapshot of the RVE are shown in Figure 4. The gravity force is not considered in the DEM part in order to be consistent with the FEM where the body force is neglected. Periodic boundary is applied to both directions of the RVE, which proves to be an optimal choice in generating most realistic stiffness for the macroscopic behaviour of granular media [43] and meanwhile satisfying a priori the Hill-Mandel-type condition [10].

\footnotetext{
¥As is known, the macroscopic friction angle of sand obtained from DEM simulation is generally smaller than that from laboratory test when spherical/circular particles are used in DEM without consideration of proper rolling resistance. In the current study, we neglect the rolling resistance in the DEM for simplicity.
} 
Table I. Parameters for the RVE.

\begin{tabular}{lccccc}
\hline Radii $(\mathrm{mm})$ & Density $\rho_{p}\left(\mathrm{~kg} / \mathrm{m}^{3}\right)$ & $E_{c}(\mathrm{MPa})$ & $v_{c}$ & Friction angle $\varphi(\mathrm{rad})$ & Damping ratio $\alpha$ \\
\hline $3-7$ & 2650 & 600 & 0.8 & 0.5 & 0.1 \\
\hline
\end{tabular}

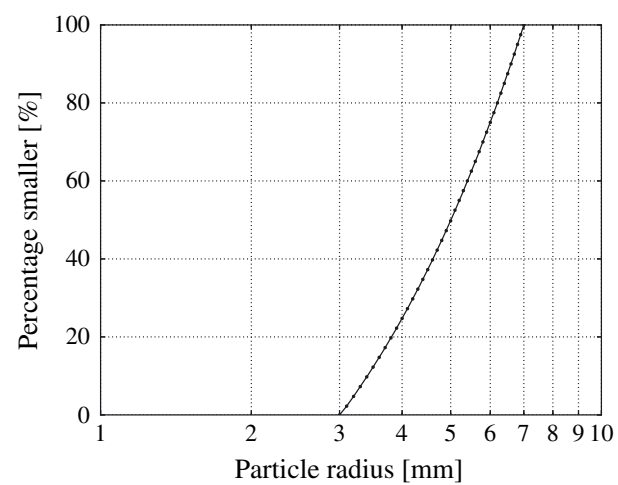

(a) PSD, percentage by particle number

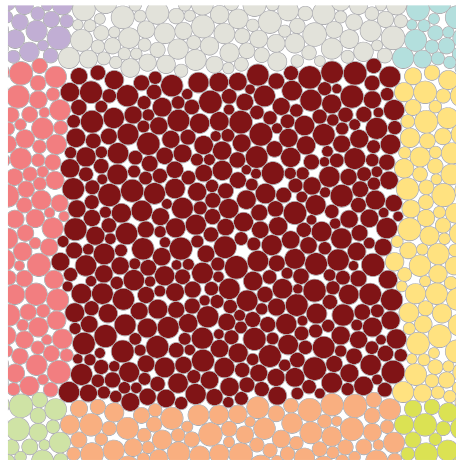

(b) RVE, periodic boundary

Figure 4. Calibrated RVE.

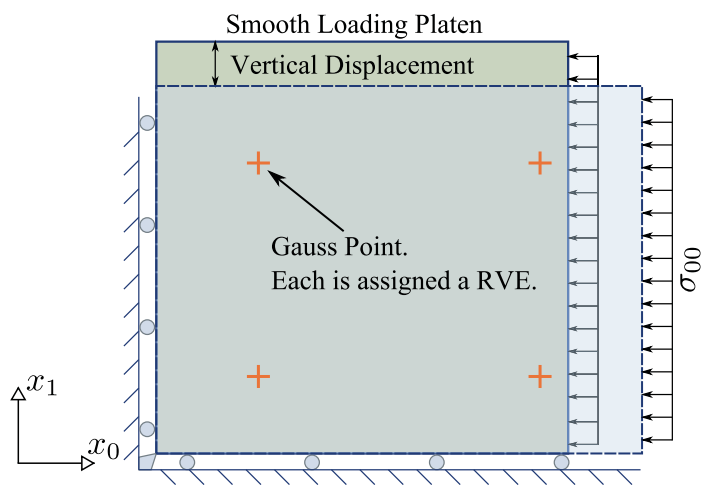

Figure 5. Depiction of the biaxial drained test with single element. The solid/dashed line shows the undeformed/deformed element, respectively.

\subsection{Single element test}

3.3.1. Macroscopic responses. A first-order quadrilateral element with four nodes and four Gauss points (thus four RVEs) is used. The element and its boundary conditions are depicted in Figure 5, where $\sigma_{00}=100 \mathrm{kPa}$ is the isotropic consolidation pressure at the initial state which is maintained constant during the shearing process. At the initial state prior to shearing, the four RVEs possess exactly the same initial condition, which leads to a uniform sample for the FEM element (with an initial void ratio 0.177). As Neumann boundary is prescribed, the SET is indeed a basic BVP for the multiscale model.

We compare the global responses of the single element test with the RVE response from a pure DEM test which has been carried out using the PeriTriaxController engine in YADE. Because the sample in SET is uniformly prepared, the multiscale tests and the pure DEM test are expected to provide very close (if not identical) responses. Indeed, this is verified by a comparison shown in Figure 6. The global vertical stress $\sigma_{11}$ of the element is calculated from the resultant force exerted on the top boundary divided by the width of the element (the thickness of the element is assumed to be unity). Similarly, $\sigma_{00}$ is the resultant lateral force on the right boundary divided by 


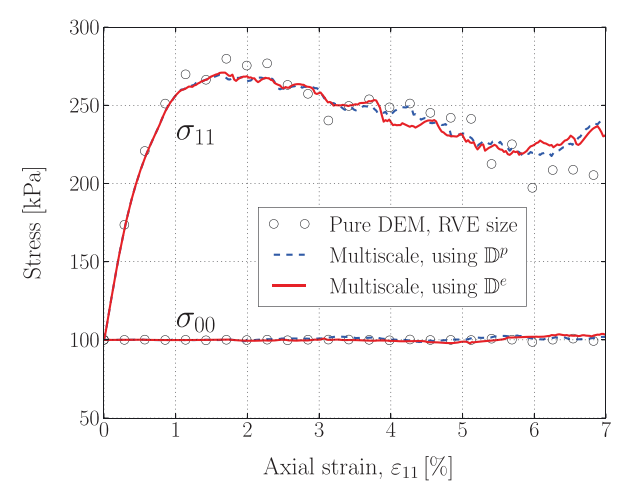

(a) Stress-strain relation

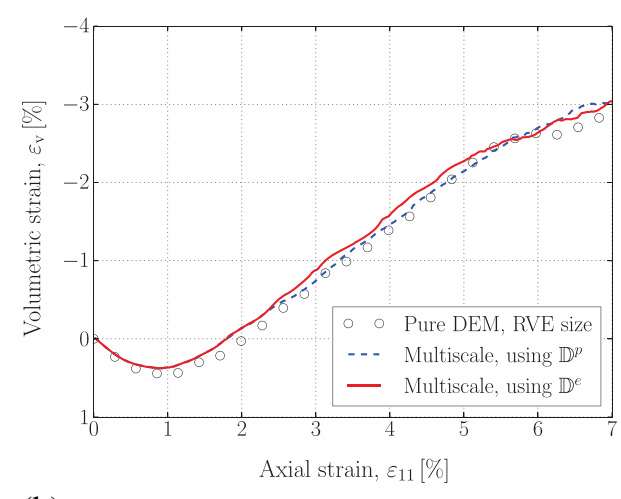

(b) Dilatancy curve

Figure 6. Macroscopic responses of the single element test. In the perturbation method, $\xi=5 \times 10^{-5}$.

the height of the element. The axial strain $\varepsilon_{11}$ and the volumetric strain $\varepsilon_{\mathrm{v}}$ are calculated from the overall deformation of the element. The figure shows that during the biaxial shear process, the multiscale modelling produces nearly identical responses with the pure DEM simulation on the RVE. The linear elasticity and the nonlinear plastic behaviour of granular media are reproduced reasonably well. The typical strain softening response and dilatancy behaviour of a medium dense sand are well captured. In particular, the use of $\mathbb{D}^{e}$ in calculating the tangent operator gives almost identical macroscopic responses with the case using $\mathbb{D}^{p}$, especially at the early stage before stress softening. Notably, there are certain deviations for both stress and volumetric strain curves between the multiscale modelling and the pure DEM modelling on the RVE, which is due to large structure change during the post-peak stress stages. The aforementioned SET benchmark verifies that the hierarchical multiscale modelling approach can faithfully reproduce the behaviour of granular media. Nevertheless, because the material responses in the multiscale approach depend crucially on the DEM simulation, careful calibration of the DEM model is required in order to match a real laboratory test. To improve the performance, one may also choose to use more complex DEM models, such as those considering rolling resistance [44], using complex-shaped particles [45] and/or employing nonlinear interparticle contact laws, albeit the calibration process may become more complicated.

3.3.2. Convergence. The performance of the multiscale approach hinges crucially on the NewtonRaphson iterative scheme employed for the global FEM solution. Because the local material behaviour is extracted from a DEM simulation that always exhibits fluctuations, we observe a linear/sublinear convergence rate in our model by using either $\mathbb{D}^{p}$ or $\mathbb{D}^{e}$ (see Figure 7). The observation is somehow different from that by Meier et al. [14], where they used the elastic modulus as the tangent operator and reported a quadratic convergence rate. Two major reasons are attributable to the difference. First, the movements of the particles in their RVEs were strictly restricted by the uniform strain field assumption (Taylor assumption), which facilitates the derivation of the elastic modulus; second, frictionless particles were used in their study, which resulted in a less plastic behaviour without energy dissipation. In our model, the local fluctuations are allowed for the particles to adjust their positions, which help to reflect the true frictional behaviour of granular media but with a trade-off of reduced convergence rate.

In conventional FEM where the constitutive relation is assumed for the prediction of the nonlinear plastic behaviour of granular media, there is also a convergence issue associated with stress integration at the material point $[46,47]$. In the current multiscale model, the nonlinear material behaviour is directly derived by DEM simulations. It hereby totally avoids the convergence issue at the material point level. Although only global errors are concerned, the following two definitions are commonly used as the criteria to judge convergence of a solution: 


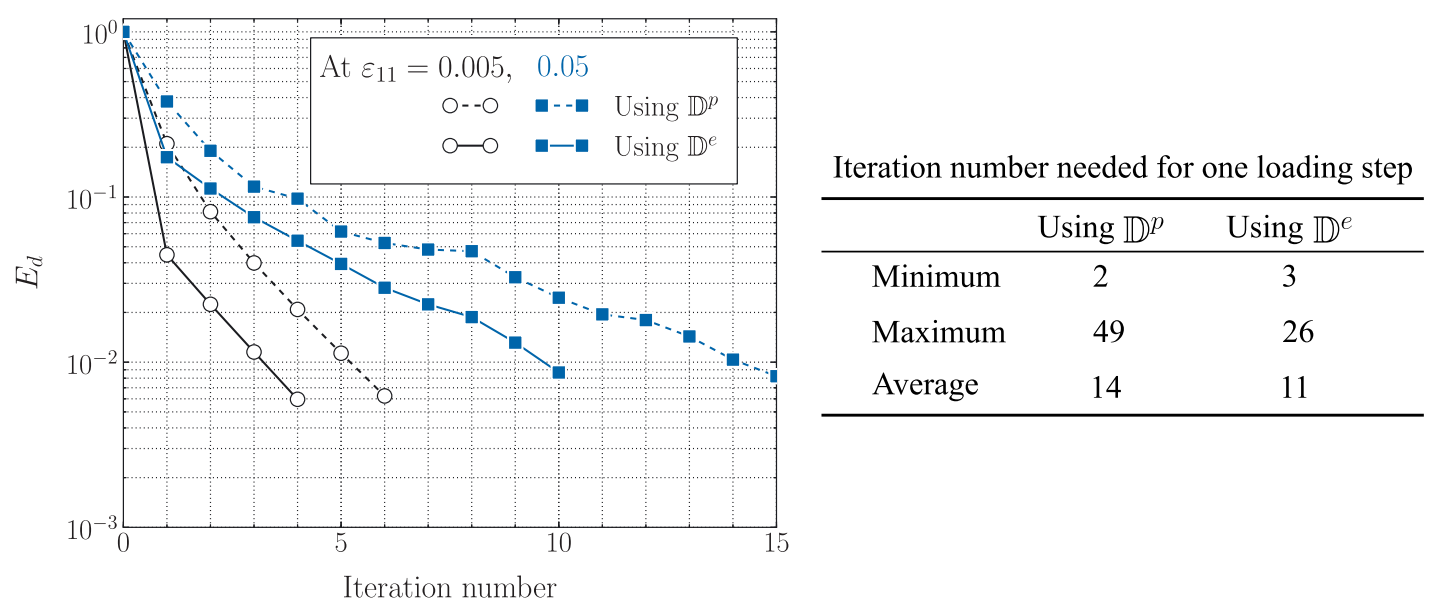

Figure 7. Comparison on convergence behaviour for different methods to calculate tangent operator.

$$
\left\{\begin{array}{l}
\text { Displacement error: } E_{d}=\frac{\left\|\boldsymbol{u}^{j}-\boldsymbol{u}^{j-1}\right\|_{2}}{\left\|\boldsymbol{u}^{j}\right\|_{2}} \\
\text { Force error: } \\
E_{f}=\frac{\left\|\boldsymbol{R}^{j}\right\|_{2}}{\|\boldsymbol{f}\|_{2}}
\end{array}\right.
$$

where the superscripts $j$ and $j-1$ refer to the two consecutive iterations within one load step and $\boldsymbol{R}^{j}$ is the residual force vector after $j$-th iteration [see Equation (4)]. $\|\bullet\|_{2}$ denotes the $L^{2}$ norm of the quantity. Here, we choose the displacement error as the criterion in this study. In practice, a tolerance of $E_{d}<0.01$ can offer satisfactory results ${ }^{\S}$ (see also [48]). Figure 7 shows a comparison of convergence based on the two methods using different modulus at two loading stages $\left(\varepsilon_{11}=\right.$ $0.5 \%$ and $5 \%$, respectively). As is shown at small strain, the converged solution can be found very fast within few iterations for both methods, whereas at large strain levels, more than 10 iterations are required. Moreover, fewer iterations are needed to find a converged solution for the method using the elastic modulus than that based on perturbation (see the summarised table in Figure 7). Indeed, the choice of the perturbation magnitude $\xi$ is too arbitrary, and in some cases, it is entirely possible that no converged solution could be found after tens of iterations. In the following tests, the method using elastic modulus will be used exclusively unless otherwise stated.

3.3.3. Parallelisation. The FEM mesh may have to be fine enough to solve a real BVP, and sometimes, higher-order element may be required to ensure adequate accuracy. Considering a DEM problem has to be solved at each of the Gauss point of the FEM mesh for EVERY iteration of the global solution, the computational cost can be horrendously high. This can be significantly relieved by parallel computing indeed. Another advantage of the current multiscale framework is the easy handling of parallelisation of the DEM simulations at each Gauss point. During every iteration and load step, each of the DEM assemblies at the Gauss points runs independently. Although the DEM computation is the most time-consuming part of the framework, it is desirable to design an effective parallelisation scheme for the DEM calculations. In a typical large-scale pure DEM simulation, the parallelisation is usually achieved by decomposing the problem domain into several subdomains $[49,50]$, where the interface particles of each subdomain need to be duplicated for information to be passed between two neighbouring subdomains. In the hierarchical multiscale framework, the consideration of domain decomposition and interfacial communication can be totally avoided because

\footnotetext{
$\bar{\S}$ A similar value is adopted as the default tolerance in some commercial softwares, for example, for global displacement|force errors: GEO5 (0.01|0.01), ANSYS $(0.05 \mid 0.005)$ and ABAQUS $(0.01 \mid 0.005)$.
} 


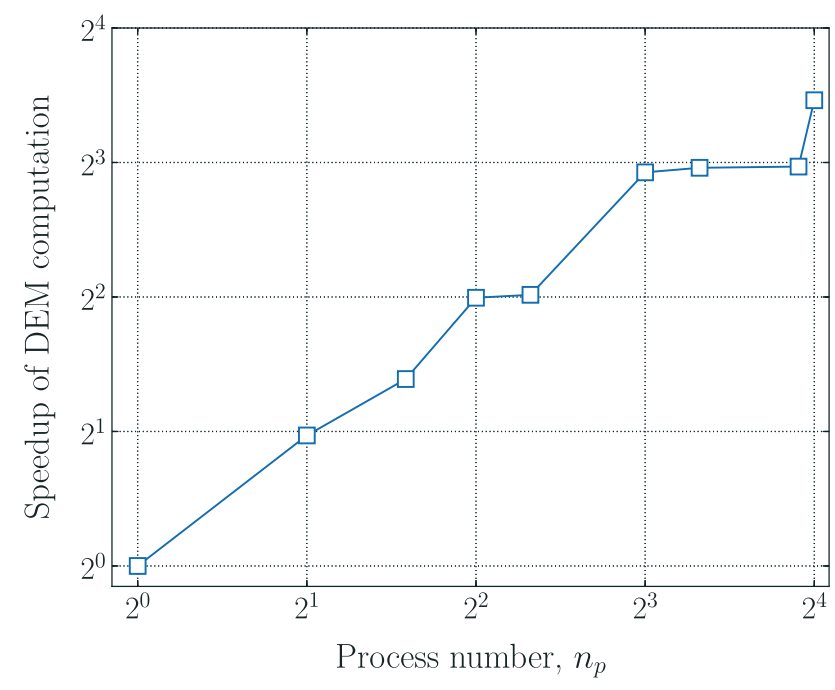

Figure 8. Speedup of DEM calculation through parallelisation.

the FEM serves naturally as a master to give instructions for these 'subdomains' (individual RVE) and to collect information from all of them.

We have enhanced our hierarchical multiscale modelling code with parallel computing capacity, by parallelising the computation of each DEM RVE at the Gauss point. To examine the scalability of our code, we run an SET with 16 Gauss points by a high-performance computing cluster with 16 identical CPU processors. We record the computational time and compare the speedups for cases using different number of processors $n_{p}$. The results are shown in Figure 8 wherein the speedup for a certain processor number is defined as the executing time using one single processor divided by that using the specific number of processors. Evidently, Figure 8 shows a clear linear scalability for the proposed hierarchical multiscale approach. The total computational time can be halved when the number of processors used are doubled. Note that it is more efficient when the computational load is evenly distributed over all used processors. For example, due to uneven distribution of computational load, the use of 9-15 processors can only achieve a similar speedup as the case using eight processors in Figure 8. Because the speedup is approximately equal to $n_{g} /\left\lceil n_{g} / n_{p}\right\rceil$, it is advisable to choose $n_{p}$ such that $n_{g}$ is divisible by $n_{p}$.

\section{MONOTONIC BIAXIAL COMPRESSION TESTS}

In this section, we employ the hierarchical multiscale framework described earlier to conduct a monotonic biaxial compression test on a $50 \mathrm{~mm} \times 100 \mathrm{~mm}$ specimen. The domain is discretised into three different meshes: coarse $(6 \times 10$ elements $)$, medium-fine $(10 \times 15$ elements $)$ and fine $(12$ $\times 20$ elements) ones. For demonstration purpose, we use the four-node quadrilateral elements with four Gauss points for all three mesh cases. An additional case using eight-node serendipity element with nine Gauss points is considered for the coarse mesh case to study the influence of element type. The FEM domain and its boundary conditions are illustrated in Figure 9. The specimen is initially uniform with all material points assigned RVEs with the same initial condition which is also same to that used in the previous SET. Based on computations using the 16-processor cluster mentioned before, the total computational time for a monotonic biaxial test amounts to around $1.4 \mathrm{~h}$ for the coarse mesh with linear elements, $3 \mathrm{~h}$ for the coarse mesh using high-order elements, also around $3 \mathrm{~h}$ for the medium-fine mesh and $4.5 \mathrm{~h}^{\mathbb{T}}$ for the fine mesh.

\footnotetext{
ISome output operations such as saving local RVE packings were omitted in this case. Otherwise, we expect a linear increase of time with the increase of number of Gauss points.
} 


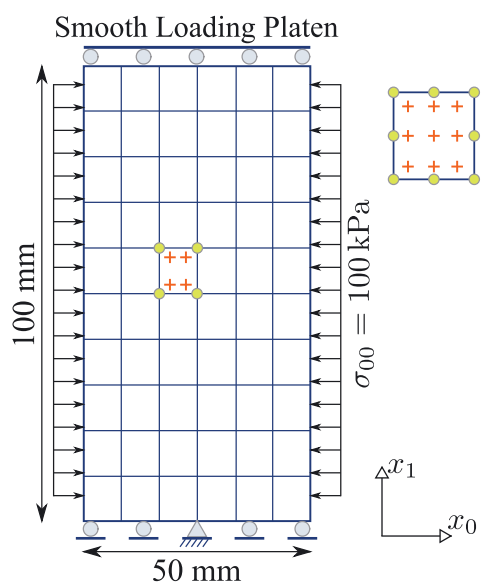

(a)

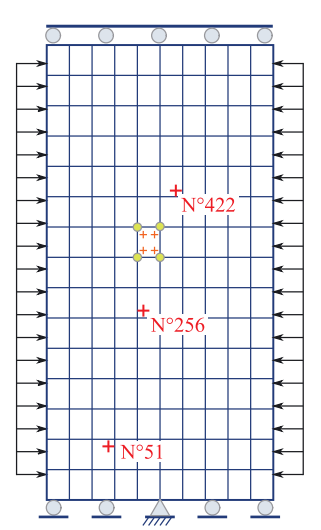

(b)

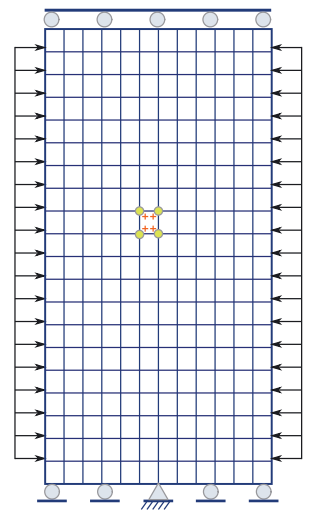

(c)

Figure 9. Discretisation and boundary condition of the specimen for monotonic biaxial compression. (a) Coarse mesh, $6 \times 10$, inset shows the supplemented high-order element; (b) medium-fine mesh, $10 \times 15$; and (c) fine mesh, $12 \times 20$. The three marked Gauss points in (b) will be used for local analyses in Section 4.2.

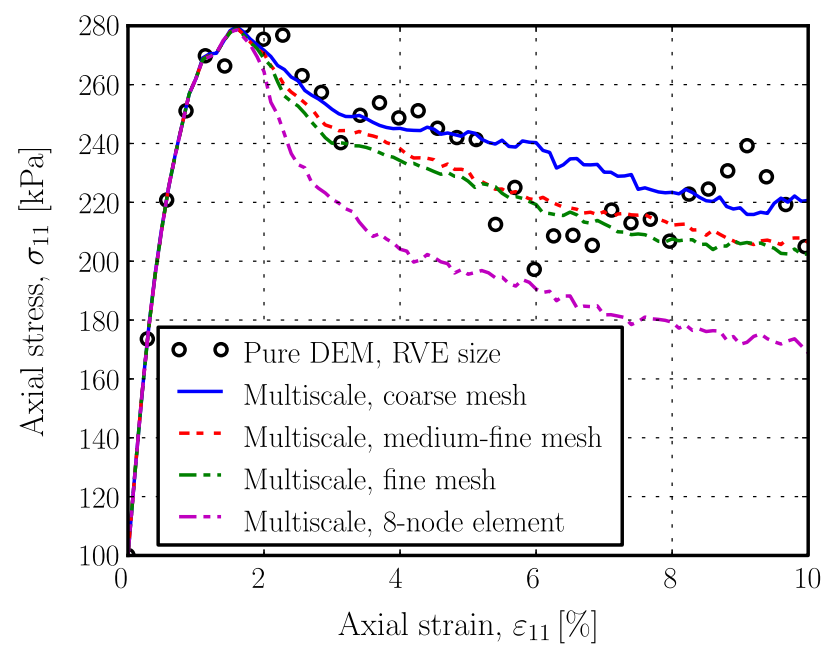

Figure 10. Global stress-strain relationship in the monotonic biaxial compression test.

\subsection{Macroscopic responses and strain localisation}

4.1.1. Global responses. The global stress-strain responses using different meshes and element types are shown in Figure 10. The response from a pure DEM test on the RVE is also presented in the figure for comparison. We first focus on the results using the lower order four-node elements, with the higher-order element case left for later discussion. As can be seen, the global responses of multiscale modelling for all three cases are generally similar to the RVE response, in particular for the pre-peak stress stage where the material behaviour is relatively elastic. Although the post-peak response of DEM test fluctuates moderately, the multiscale modelling results are rather smooth. All three cases of FEM mesh yield an identical pre-peak stress behaviour, but their post-peak responses bifurcate from one another. Although they all show a softening post-peak trend, the finer meshes lead to more significant softening behaviour and smoother curve than the coarse case. Indeed, the medium-fine and fine mesh cases result in rather close global responses already, which partially prove the consistency of the proposed framework in terms of mesh density. Mesh size dependency is commonly observed in FEM simulation of strain localisation. Typically, the use of finer mesh 
leads to more concentrated strain and narrower shear band as well as softer global responses. The same phenomenon is observed in the current multiscale simulations as will be discussed in the following sections.

4.1.2. Breaking symmetry and strain localisation. Strain localisation is a natural phenomenon observed in many materials including granular media. In civil engineering, strain localisation in sand is believed to be a precursor of many catastrophic hazards such as landslides, debris flow and failure of relevant geostructures. Modelling of strain localisation has been an active area by researchers using both FEM and DEM. How the material response breaks symmetry and triggers strain localisation has been a particularly interesting issue in relevant studies. In laboratory test on sand or in DEM simulations with symmetric smooth boundary condition, strain localisation can be observed due to the inherent inhomogeneity of the material. However, in traditional FEM simulations, one needs to add artificial imperfection(s) or use random distributed local properties to trigger strain localisation when symmetric smooth boundary conditions are applied [51-53]. Recently, Gao and Zhao [7] showed that strain localisation could occur in a uniform sample with smooth, symmetric boundary conditions based on a sand plasticity model accounting for fabric and its evolution that can naturally produce non-coaxial material response for a granular media [3-6]. For a sand sample subject to biaxial shear, Gao and Zhao [7] further demonstrated that a single shear band will occur in the smooth, symmetric boundary condition case, and the non-coaxial material response plays a role of symmetry breaker to trigger the strain localisation. Shown in Figure 4 of their paper, Gao and Zhao [7] showed that the displacement field is indeed not symmetric and is inclined in one direction (depending on the angle between applied strain increment and initial fabric anisotropy) even when the boundary condition is smooth and symmetric.

It is interesting to investigate the same issue by our multiscale approach. Figure 11 shows the initial nodal displacement field of the sample based on the fine mesh results. Although the sample is uniform and the boundary conditions are symmetric and smooth, it is interesting to see that

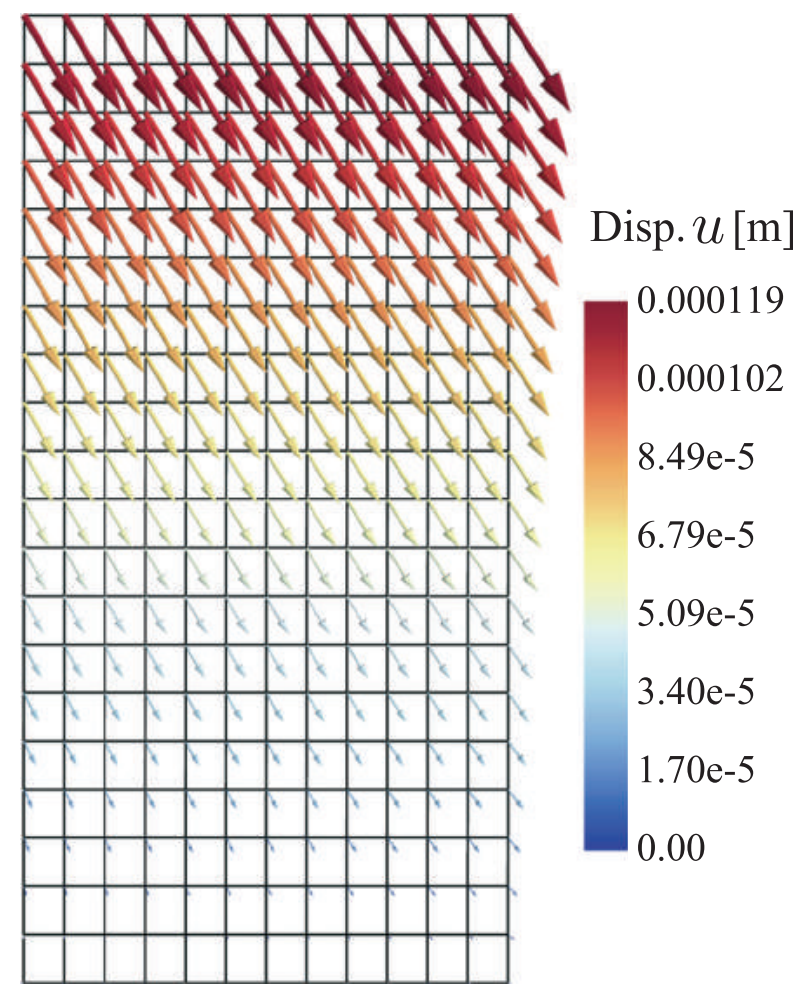

Figure 11. Nodal displacement field of the sample at the very beginning of compression $\left(\varepsilon_{11}=0.1 \%\right)$ using the fine mesh. 
the displacement field from our simulation displays a similar non-symmetric pattern inclined to one direction as shown in [7], (the arrows are parallel but have different displacement magnitudes proportional to the nodal heights, which suggest a uniform deformation field). This is because the RVE used in the multiscale model is a disordered packing that can naturally capture the non-coaxiality of the material in terms of the tangent operator (using either $\mathbb{D}^{p}$ or $\mathbb{D}^{e}$ ) in Equation (3). As seen from Figure 3, the RVE packing with 400 particles after isotropic consolidation still possesses a small degree of anisotropy in terms of the distribution of contact normals. This tiny anisotropy provides an important inducing factor on symmetry breaking. Strain localisation can therefore be triggered in the sample, as shown in Figure 12, without bothering to introduce any artificial imperfection or random field. The localisation patterns using different meshes are quite similar in Figure 12. The localisation intensities are, however, different for different mesh densities and types (note the colour ranges are different in different subfigures). At the global peak stress state $\left(\varepsilon_{11}=1.6 \%\right)$, the fields of accumulated deviatoric strain and void ratio remain relatively homogeneous. The minimum $\varepsilon_{q}$ is around $0.26-0.27$ and the maximum value around $0.32-0.33$. The void ratio field is even more uniform with values ranging from 0.173 to 0.174 in all three cases. Despite the relative uniformity, a clear trend for strain localisation can be observed due to the inclined displacement field developed from the very beginning of compression. Indeed, at the state when $\varepsilon_{11}=10 \%$, an overwhelmingly localised shear band is formed in all three cases. The localised region largely corresponds to the most dilative zone with maximum void ratio, which is consistent to the results in traditional FEM simulations as well as experimental observations [54]. However, the concentrated band of void ratio is found generally wider than that of the deviatoric strain. This is because the dilative volumetric strain will reach saturation after extensive shear deformation develops within the localised area and when the critical state is reached.

4.1.3. Mesh/element type dependencies. As mentioned earlier, the macroscopic response of the sample, in particular the post-peak strength, depicts an apparent feature of mesh dependence. In addition, the localised behaviour in the sample is also dependent on the mesh as well as the element type. As seen from Figure 12, a finer mesh generally gives rise to more concentrated and higher localised deviatoric strain as well as void ratio. For example, the maximum $\varepsilon_{q}$ and void ratio in the fine mesh case are 0.809 and 0.258 , respectively, whereas their values are only 0.507 and 0.234 , respectively, in the coarse mesh case. This is consistent with the observation in Figure 10 that the use of finer mesh generally leads to a softer post-peak response. Although no intrinsic length scale has been considered in the multiscale approach, the width of shear band observed from our study shows an apparent mesh dependency. As is seen in Figure 12, the localised shear band is wider when the coarse mesh is used, whereas it is narrower with a finer mesh. Meanwhile, we find that the influence of the element type is even more obvious. We notice that the pre-peak stress state behaviour of higher-order element case is identical with the linear element cases as shown in Figure 10 and the contours of accumulated deviatoric strain and void ratio at the peak stress state for the high-order element case are also similar to those using low-order elements [see Figure 12(g)]. Although the post-peak stress state response in the high-order element case is softer compared with those using low-order ones, a higher concentration of deviatoric strain is observed shown in Figure 12(h), where the largest accumulated deviatoric strain $\varepsilon_{q}$ reaches $124.9 \%$, a value more than 1.5 times larger than that in the case using the fine mesh. The shear band width is also much narrower than all the other three lower-order element cases. The observations indicate that the property of mesh/element type dependencies in the multiscale solution is indeed inherited from the conventional FEM approach at the macroscopic level. Depending on the nature of the problem to be treated, lower-order elements could be sufficient such as in the design of some geostructures where pre-peak stress states are more concerned (to prevent failure), whereas in other cases requiring high accuracy, high-order elements may be considered. Here, as our major interest is placed on the framework itself rather than the accuracy of its solution to a particular problem, the following analyses are exclusively based on using the four-node isoparametric element. 

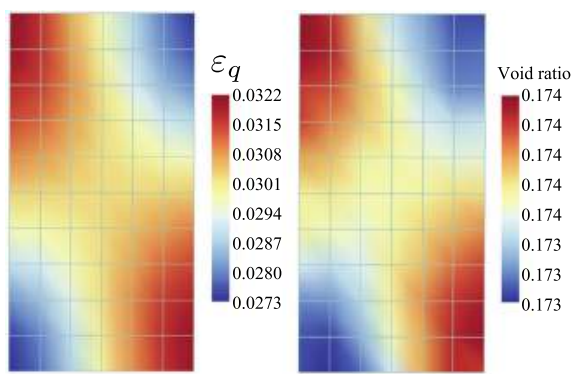

(a) Peak stress state, $\varepsilon_{11}=1.6 \%$
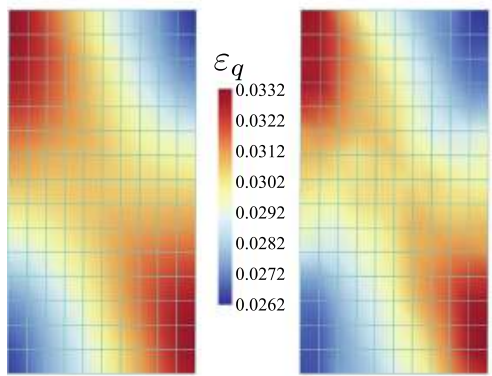

(c) Peak stress state, $\varepsilon_{11}=1.6 \%$
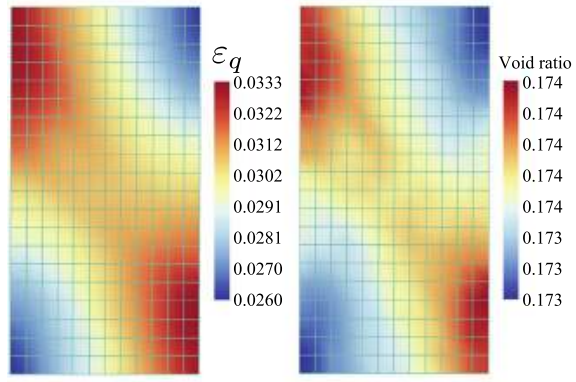

(e) Peak stress state, $\varepsilon_{11}=1.6 \%$
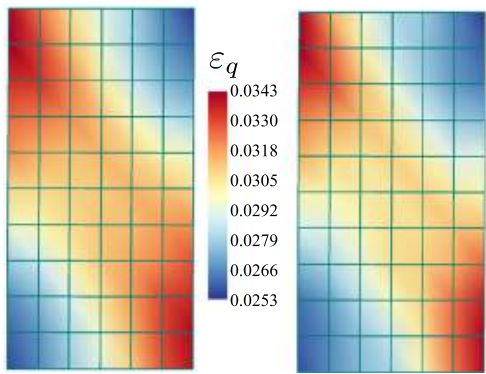

(g) Peak stress state, $\varepsilon_{11}=1.6 \%$

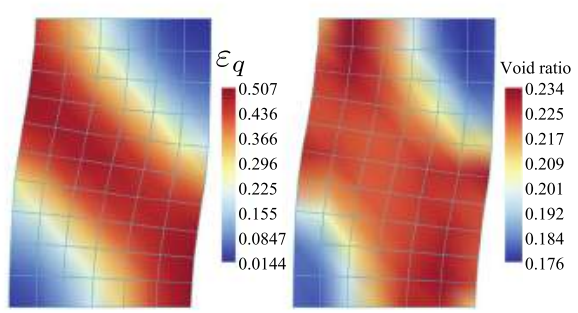

(b) Final state, $\varepsilon_{11}=10 \%$

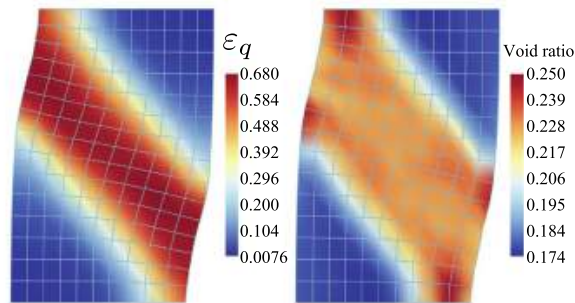

(d) Final state, $\varepsilon_{11}=10 \%$
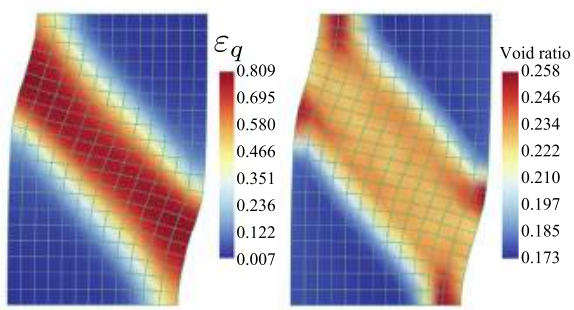

(f) Final state, $\varepsilon_{11}=10 \%$

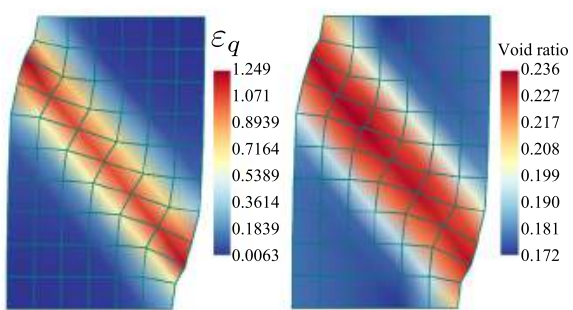

(h) Final state, $\varepsilon_{11}=10 \%$

Figure 12. Contours of the accumulated deviatoric strain and void ratio showing strain localisation. Note that different colour ranges are used in different subfigures to show the localised patterns. (a) and (b) coarse mesh; (c) and (d) medium-fine mesh; (e) and (f) fine mesh; (g) and (h) coarse mesh with eight-node elements.

4.1.4. RVE packing and strain localisation. To further study the influence of the RVE packing (disordered versus regular) on the occurrence of strain localisation, we prepare a regular hexagonal packing and embed it as the RVE into the coarse FEM mesh. Due to its lattice-like structure, the pure 
DEM response exhibits a rather high stiffness and shows a linear elastic behaviour at the early stage of compression. Although after reaching the peak stress (which is about five times higher than that using the disordered RVE) at around $\varepsilon_{11}=2.8 \%$, the strength of the material drops abruptly, shown as open circles in Figure 13. The multiscale model well captures the extremely brittle response of the material, showing a linear elasticity coinciding with the pure DEM simulation up to peak then followed by an abrupt decrease of strength (with a nearly vertical drop from $\mathcal{A}$ to $\mathcal{B}$ in Figure 13). The post-peak behaviour of the pure DEM response (almost constant stress) is also well captured by the multiscale simulation.

Another interesting finding is the deformation field at the peak stress state (Point $\mathcal{A}$ ), which is rather homogeneous with the deviatoric strain ranging from 0.0303 to 0.0305 , due to the use of regular RVE. No obvious trace of strain localisation is observed at this stage, which is quite different from the cases using disordered RVE as shown in Figure 12. However, a sudden strain localisation is found accompanying with the drastic drop of strength within only one global loading step $\left(\Delta \varepsilon_{11}=\right.$ $0.1 \%$, Point $\mathcal{B}$ ). The accumulated nodal displacement fields of the sample at the two consecutive loading stages $\mathcal{A}$ and $\mathcal{B}$ are shown in Figure 14. At Point $\mathcal{A}$, the displacement field is symmetric, resulting in a uniform deformation field, and all RVE packings maintain their original regular latticelike structure [Figure 14(a) shows a RVE packing at the location of the FEM mesh with maximum deviatoric strain]. This means that the stress-strain response of the regular RVE is coaxial at Point

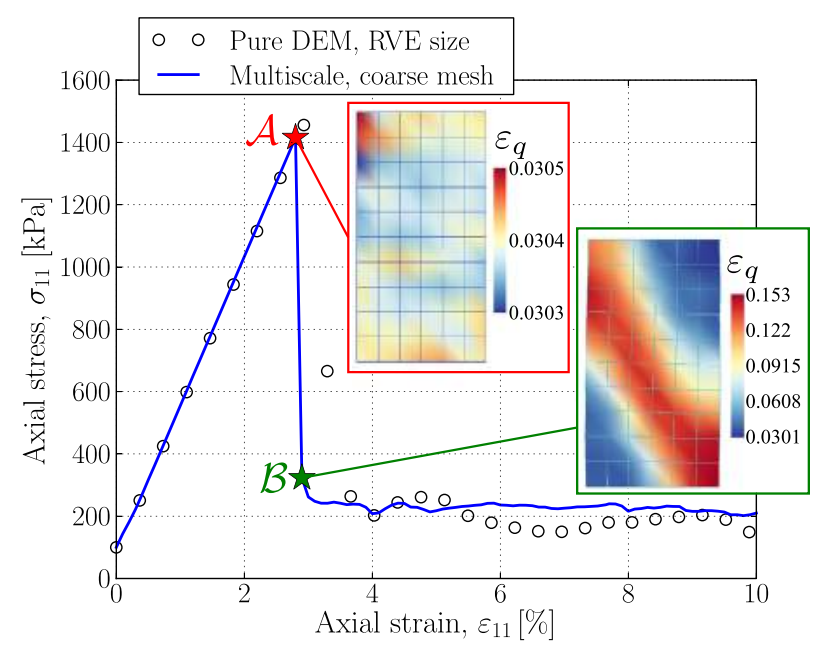

Figure 13. Macroscopic response of the multiscale model using a regularly packed RVE and the coarse mesh.

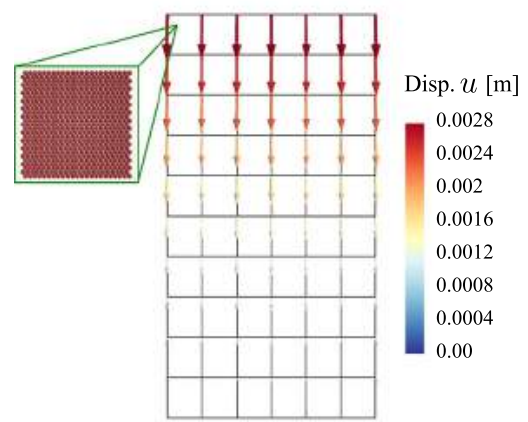

(a) Point $\mathcal{A}, \varepsilon_{11}=2.8 \%$

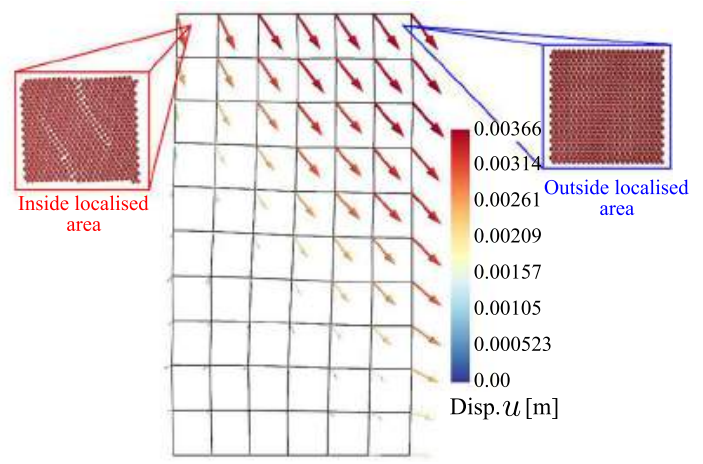

(b) Point $\mathcal{B}, \varepsilon_{11}=2.9 \%$

Figure 14. Accumulated nodal displacement fields at Points $\mathcal{A}$ (peak state) and $\mathcal{B}$ (break state) in Figure 13 and the structures of some typical RVEs. 
$\mathcal{A}$, which in theory will not induce strain localisation under smooth, symmetric boundary condition. However, at Point $\mathcal{B}$, the symmetry of the displacement field suddenly breaks up, forming an obvious slip line within the sample that causes the localisation of the strain shown in Figure 13. A further scrutiny of the RVE packing inside the localised area indicates that it loses its initial structural regularity with two cracks appearing in the packing. This confirms that the breaking of regularity of the RVE at large deformation results in the non-coaxial response in the material, which in turn leads to the formation of strain localisation at Point $\mathcal{B}$. Meanwhile, it is found that the RVE packings outside the localised area maintain the lattice-like structure during the loading course.

\subsection{Local response and microscopic characteristics}

As mentioned, the hierarchical multiscale model has the advantage to solve engineering level BVPs and meanwhile to provide insights into the behaviour of microstructure at locations of interest in the macroscopic domain. As an indicator of the microstructure in sand, soil fabric has been commonly used to characterise the microscopic behaviour in a granular material. Here, we employ a popular contact normal-based tensorial definition on fabric following Satake [55] and Oda [56]

$$
\boldsymbol{\phi}=\int_{\Theta} E(\Theta) \boldsymbol{n}^{c} \otimes \boldsymbol{n}^{c} \mathrm{~d} \Theta=\frac{1}{N_{c}} \sum_{N_{c}} \boldsymbol{n}^{c} \otimes \boldsymbol{n}^{c}
$$

where $\Theta$ denotes the orientation of $\boldsymbol{n}^{c}$ in the global coordinate system, the probability distribution function $E(\Theta)$ of the contact normals can be approximated to a second-order Fourier expansion (in 2D)

$$
E(\Theta)=\frac{1}{2 \pi}\left[1+\boldsymbol{F}_{a}:\left(\boldsymbol{n}^{c} \otimes \boldsymbol{n}^{c}\right)\right]
$$

where the deviatoric tensor $\boldsymbol{F}_{a}$ quantifies the fabric anisotropy (note $\operatorname{tr} \phi=1$ )

$$
\boldsymbol{F}_{a}=4 \times \operatorname{dev} \boldsymbol{\phi}=4\left(\boldsymbol{\phi}-\frac{1}{2} \boldsymbol{\delta}\right)
$$

The second invariant of $\boldsymbol{F}_{a}, F_{a}$, has been commonly used to measure the anisotropic intensity

$$
F_{a}=\sqrt{\frac{1}{2} \boldsymbol{F}_{a}: \boldsymbol{F}_{a}}
$$

4.2.1. Local responses of material points inside/outside the shear band. We choose the three Gauss points as marked in Figure 9(b) for the medium-fine mesh case to examine the local response and the evolution of fabric anisotropy during the loading and shear banding process. Note that $\mathrm{N}^{\circ} 51$ is located far away from the shear band, $\mathrm{N}^{\circ} 256$ is inside the shear band and $\mathrm{N}^{\circ} 422$ is just located at the edge of the shear band. The local responses of the respective RVEs at the three Gauss points are depicted in Figure 15.

(i) $N^{\circ} 51$. Because $\mathrm{N}^{\circ} 51$ is far away from the localised zone, it only develops a rather small strain to $\varepsilon_{q}=2.8 \%$ before undergoing an unloading due to the strain concentration within the shear band. The unloading process is clearly seen from Figure $15(\mathrm{a}-\mathrm{c})$ where we observe a reversed $\varepsilon_{q}$ accompanied by apparent drop in both $q$ and fabric anisotropy $F_{a}$. The volume change of this point is also very small, with a maximum contraction $\varepsilon_{\mathrm{v}}=0.46 \%$. The stress path at $\mathrm{N}^{\circ} 51$, shown in Figure 15(b), indicates that the point undergoes a perfect drained loading path as the slope of the stress path is $1: 1$ in $2 \mathrm{D}$ tests (it is $q / p=3: 1$ for drained conventional triaxial compression tests). 


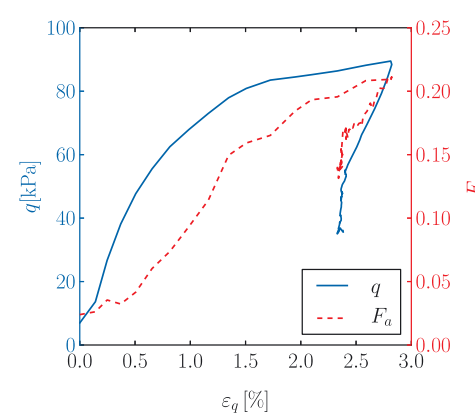

(a) Evolution curve

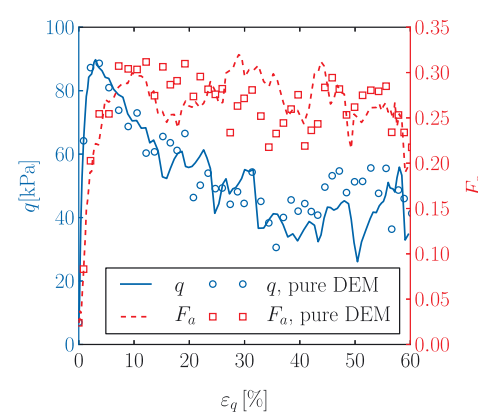

(d) Evolution curve

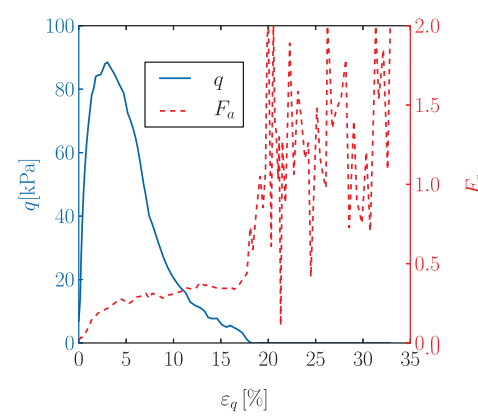

(g) Evolution curve

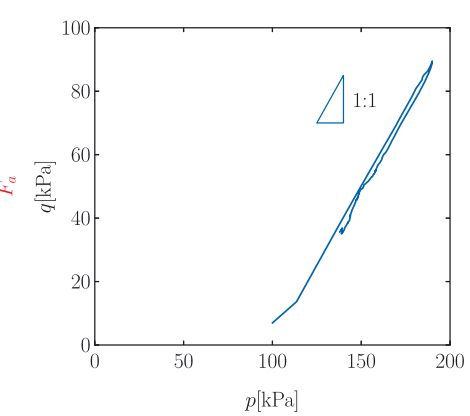

(b) Loading path

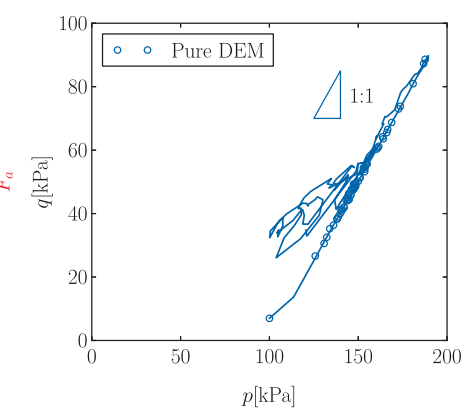

(e) Loading path

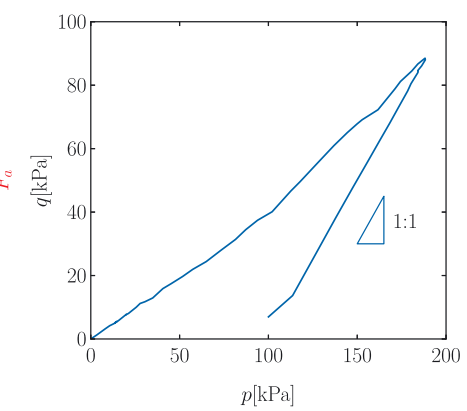

(h) Loading path

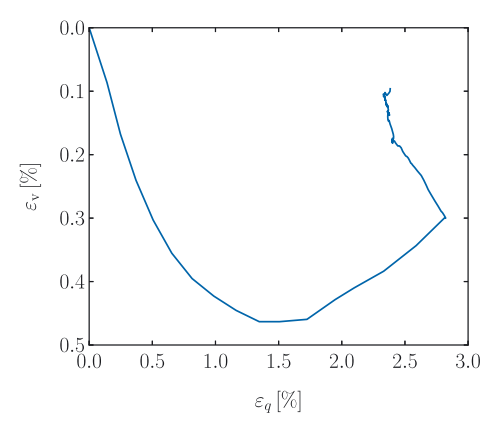

(c) Dilatancy curve

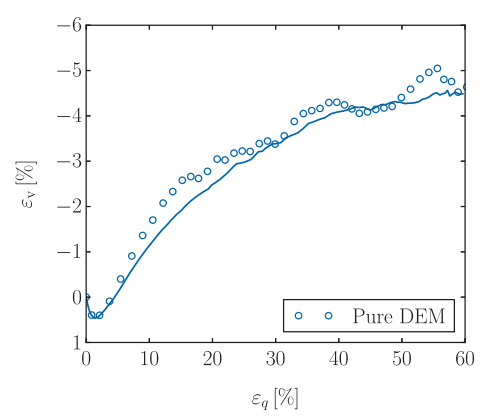

(f) Dilatancy curve

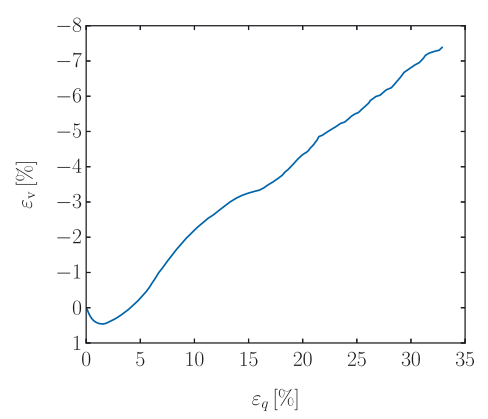

(i) Dilatancy curve

Figure 15. Local responses of the three selected Gauss points during the test using the medium-fine mesh. $(\mathrm{a}-\mathrm{c}) \mathrm{N}^{\circ} 51 ;(\mathrm{d}-\mathrm{f}) \mathrm{N}^{\circ} 256 ;(\mathrm{g}-\mathrm{i}) \mathrm{N}^{\circ} 422$.

(ii) $N^{\circ} 256$. The material responses at $\mathrm{N}^{\circ} 256$ are quite different from that at $\mathrm{N}^{\circ} 51$. Located inside the shear band, $\mathrm{N}^{\circ} 256$ undergoes a monotonic loading process up to a very large strain level $\varepsilon_{q}=60 \%$. The local responses of this point are much more fluctuated than that at $\mathrm{N}^{\circ} 51$ and resembles to those obtained from a pure DEM test as shown in Figure 10. Because the stress path for $\mathrm{N}^{\circ} 256$ matches reasonably well with that of a monotonic drained loading path, the responses of $\mathrm{N}^{\circ} 256$ are further compared with that obtained from a separate pure DEM test on the RVE packing subject to monotonic-drained biaxial compression using the PeriTriaxController engine in YADE. The pure DEM simulation results are shown as open symbols in Figure 15(d-f). It is evident that the two are rather consistent with each other. We also notice that after sustained shear, $\mathrm{N}^{\circ} 256$ reaches the so-called critical state $[57,58]$ with constant $q, F_{a}$ and $\varepsilon_{\mathrm{v}}$ (albeit with strong fluctuations). As the initial RVE has been prepared to be a dense sample, this material point reaches a maximum dilatancy $\varepsilon_{\mathrm{v}}=-4.5 \%$ at critical state. The observation is consistent with the experimental finding $[59,60]$ as well as DEM simulations that critical state can be reached within the shear band [61]. 


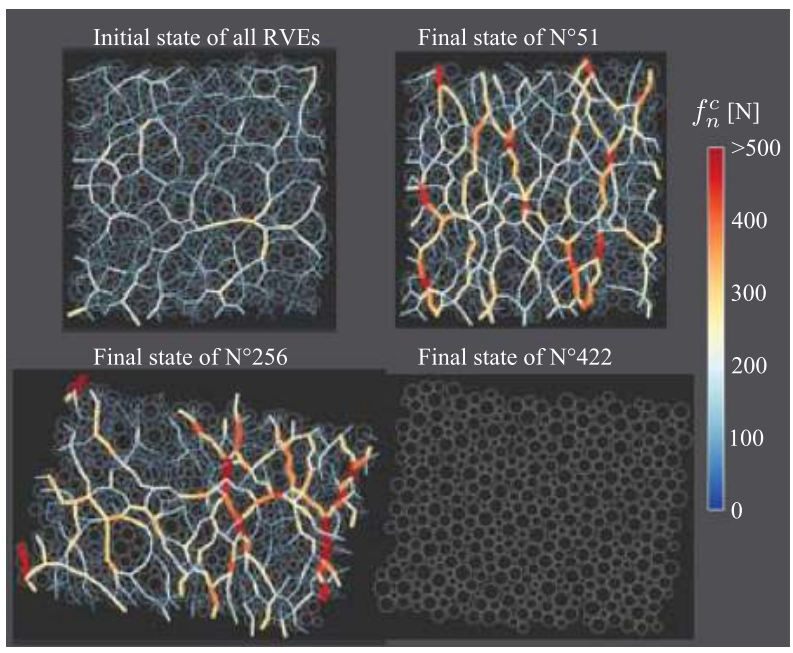

(a)

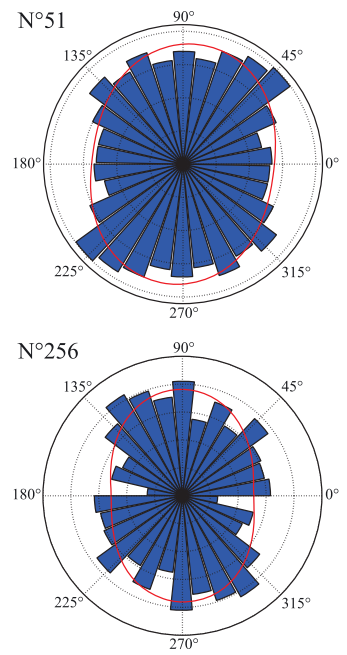

(b)

Figure 16. (a) The structure and force chains of the local initial and deformed RVEs and (b) the corresponding distributions of the contact normals in $\mathrm{N}^{\circ} 51$ and $\mathrm{N}^{\circ} 256$. The smooth red curves in (b) are the Fourier approximations using Equation (23).

(iii) $N^{\circ} 422$. Located at the boundary of the localised zone, $\mathrm{N}^{\circ} 422$ shows quite different responses than the previous two points do. It experiences a monotonic loading path until a relatively large strain level $\varepsilon_{q}=33 \%$. Whilst right after the peak stress state at $\varepsilon_{q}=3 \%, \mathrm{~N}^{\circ} 422$ undergoes a partially drained loading path, where the slope of the loading path in Figure 15(h) is less than 1:1 and the mean pressure $p$ decreases much faster than deviatoric stress $q$ does. As both $p$ and $q$ evolve towards zero, at around $\varepsilon_{q}=18 \%$, the material point totally loses its strength, which leads to a local failure for this material point. Because the packing is now possessing very few contacts at the zero stress state, the contact normal-based fabric tensor cannot be appropriately defined, which explains the unrealistic great fluctuation of $F_{a}$ after the failure in Figure $15(\mathrm{~g})$. It is noteworthy that the failure mode for $\mathrm{N}^{\circ} 422$ is different from liquefaction as the loading path does not follow an undrained one but a partially drained one to the failure point. Nevertheless, $\mathrm{N}^{\circ} 422$ fails due to unconfined dilation as seen from Figure 15(i) where the maximum dilatancy for the packing reaches $\varepsilon_{\mathrm{v}}=-7.4 \%$. When the volume of the material unrestrictedly expands, the contact number steadily decreases, which leads to total failure of the packing. It is hence interesting to see that even though globally the FEM sample still has the capacity to sustain external loading (even during the softening stage in Figure 10), locally, there may be some points that totally lose their strength already. Physically, these material point may correspond to certain cavities created during the shearing and localisation processes.

4.2.2. Fabric structure and local failure. Figure 16(a) shows a direct comparison of the contact force network for the three RVE packings at the initial (after consolidation) and final $\left(\varepsilon_{11}=10 \%\right)$ states where the contact force chains are superimposed with the granular assemblies by connecting the centroids of the particles at every contact. The width of the force chain is proportional to the magnitude of the interparticle contact normal force (see also the colour map). All RVEs possess the same initial condition which is relatively isotropic with no obvious preferably orientated strong force chains. At the final state, the DEM packing $\mathrm{N}^{\circ} 51$ does not experience much deformation as its configuration (in terms of particle positions) is very similar to that at the initial state. Nevertheless,

\footnotetext{
"Here, failure means the total loss of strength. $F_{a}=2$ corresponds to the extreme case of all contact normals in a packing point to a same direction.
} 


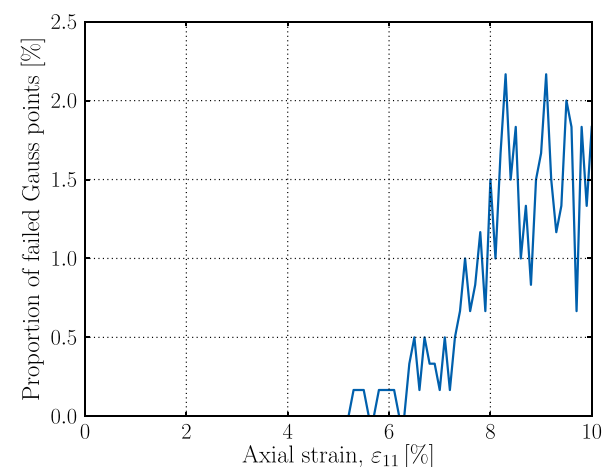

(a)

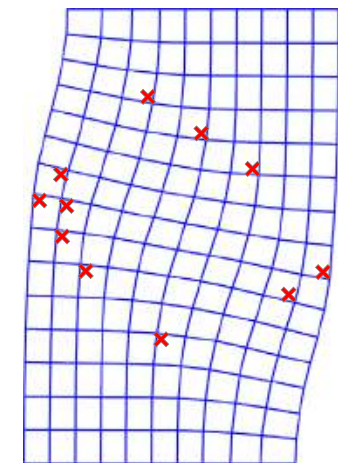

(b)

Figure 17. (a) Proportion of failed Gauss points during the compression test using the medium-fine mesh and (b) their distribution over the domain at the final state.

several distinct strong force chains are observed which are parallel to the vertical compression direction. For $\mathrm{N}^{\circ} 256$, the deformation is considerably large with a severely deformed configuration as compared with the initial state (also with noticeable rotation). The structure of the packing is also more heterogeneous, and strong force chains are more concentrated to two major penetrating ones. The maximum contact normal force is also much larger than that in $\mathrm{N}^{\circ} 51$. Although the packing is highly distorted, the two major strong force chains are found still aligning along the vertical compression direction, acting as the supporting columns to the deviatoric shear stress. The packing of $\mathrm{N}^{\circ} 422$ also deforms noticeably. Due to severe volumetric expansion, however, the packing becomes much dilute wherein no apparent force chain is observable. Note that the deformation gradients of $\mathrm{N}^{\circ} 256$ and $\mathrm{N}^{\circ} 422$ are consistent with the global shear band inclination direction. The contact normal distribution shown in Figure 16(b) further confirms that the major principal directions for both $\mathrm{N}^{\circ} 51$ and $\mathrm{N}^{\circ} 256$ are close to the vertical compression direction. As there exists local failure, it is interesting to monitor the occurrence, location, and proportion of failed local packings amongst all Gauss points in the FEM sample. Shown in Figure 17(a), the local failure firstly occurs at $\varepsilon_{11}=5.3 \%$. Then the proportion of failed points gradually increases to an average value of $1.5 \%$ which means about 10 out of 600 Gauss points totally lose their strength. These failed points are found mainly located along the edges of the shear band, as shown in Figure 17(b). A further comparison with Figure 12(d) indicates that the locations of these failure material points are coincident with those experiencing high dilations (with large void ratios).

4.2.3. Concentration of fabric anisotropy. Based on simulation by their enhanced model considering fabric and its evolution, Gao and Zhao [7] showed that fabric anisotropy reaches its maximum inside the shear band. In their model, the void vector-based fabric tensor definition is assumed to be a monotonic increasing function of the plastic shear strain. DEM studies indicate that the contact normal-based fabric tensor used here may decrease upon shearing during the softening stage [62, 63]. In Figure 18, we show the contours of the fabric anisotropic intensity over the whole domain at two stages of our multiscale simulation of the fine mesh case: $\varepsilon_{11}=4 \%$ and $\varepsilon_{11}=4.8 \%$. Although the contour at $\varepsilon_{11}=4 \%$ shows a localised pattern similar to that of the deviatoric strain or the void ratio, the contour at $\varepsilon_{11}=4.8 \%$ depicts a pattern with concentrated fabric anisotropy along the edges of the shear band and slightly less intensity in the middle (but still greater than that at $\varepsilon_{11}=4 \%$ ). A further inspection indicates that the concentrated regions along the shear band edges coincide indeed with the local material points approaching failure for the fine mesh case [c.f., Figure 17(b) for a medium-fine mesh case]. Due to severe dilation at these local points, the contact numbers drop significantly with dramatic jumps in fabric anisotropy (the maximum value almost doubles). At even later stage $\left(\varepsilon_{11}>5.3 \%\right)$, the contact normal-based fabric anisotropy is no longer computable because the packings at these locations fail totally and there are no interparticle 


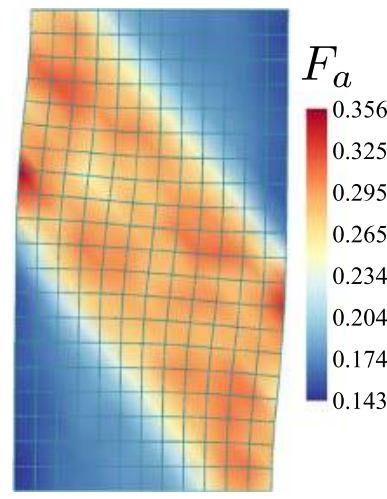

At $\varepsilon_{11}=4 \%$

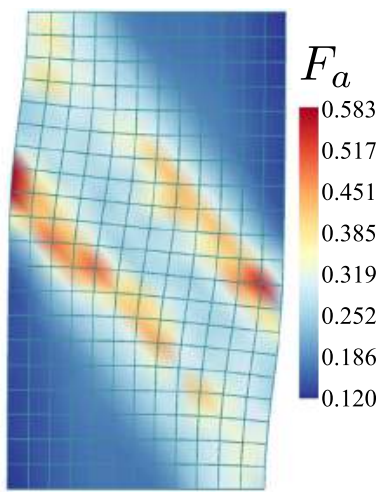

At $\varepsilon_{11}=4.8 \%$

Figure 18. Contour of fabric intensity at $\varepsilon_{11}=4 \%$ and $\varepsilon_{11}=4.8 \%$ in the test using the fine mesh.

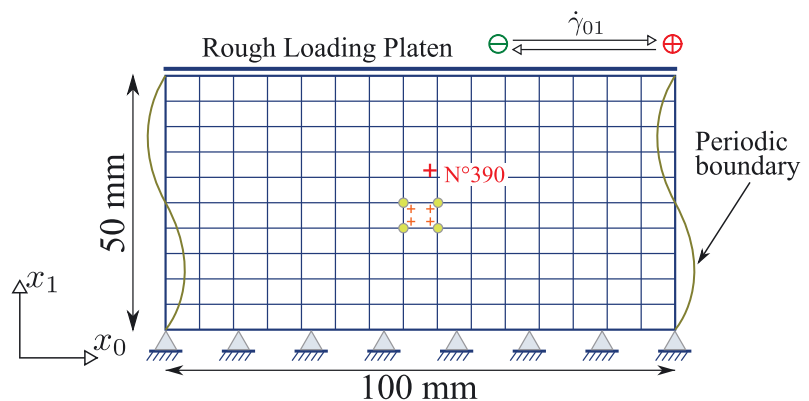

Figure 19. Discretisation $(15 \times 10$ elements $)$ and boundary condition of the specimen for cyclic simple shear. The $\mathrm{N}^{\circ} 390$ Gauss point will be used for local analyses.

contacts in these RVEs. We hereby recommend the accumulated deviatoric strain and the void ratio as good indicators for the identification of strain localisation but not the fabric anisotropy (at least not the contact normal-based one).

\section{CYCLIC SIMPLE SHEAR TESTS}

Accurately modelling the cyclic behaviour of granular media is known to be particularly challenging. In conventional continuum modelling, special constitutive models (e.g. using bounding surface) have to be developed to capture the hysteresis behaviour of the material subject to cyclic loading. We will demonstrate in the following that the hysteresis behaviour can be naturally reproduced by the current multiscale model. We employ two examples of cyclic simple shear tests to showcase this capability of the proposed model. The sample to be sheared has a dimension of $100 \mathrm{~mm} \times 50 \mathrm{~mm}$, which is discretised by a medium-fine FEM mesh $(15 \times 10$ elements $)$. The boundary condition is shown in Figure 19 where the bottom surface is totally fixed and the top surface is sheared horizontally via a rough loading platen which yields a constant volume undrained shearing condition for the specimen. The left and right boundaries are periodic to reduce the boundary effects. The same RVE as shown in Figure 4 is used here but is prepared with a different initial void ratio. In the previous monotonic biaxial compression, we prepare the sample in a dense state for easier formation of shear band accompanying with stress softening. Here, we prepare a loose initial state for the RVE in order to observe potential liquefaction during the cyclic loading. Two commonly used control modes are simulated: the first one corresponds to a maximum shear strain controlled testing mode; the second one is controlled by a maximum shear stress. In both cases, the model responses are similar to those 
from laboratory tests [64] and other model simulations [65]. The hysteresis loop is well captured, and liquefaction or cyclic mobility can be defined accordingly.

\subsection{Maximum shear strain controlled test}

A uniform (i.e. using the same RVE for all Gauss points) and loose (with an initial void ratio 0.223) sample is considered. Subject to maximum shear strain-controlled test, the global stressstrain response and the loading path of the sample are shown in Figure 20. The responses reproduce qualitatively well the experimental observations on sand [64] and are consistent with other model simulations [65] of cyclic simple shear tests as well. This initially loose sample shows a contractive response with steady decrease in effective stress (both the normal pressure and the peak shear stress). The peak shear stress in the negative loading direction (sign definition is given in Figure 19) is always smaller than that in the positive loading direction due primarily to the fabric rearrangements of the granular media. The simulation is terminated after 12 cycles of shear when the final stress is extremely low, that is, $\sigma_{11}=8.6 \mathrm{kPa}$ and $\sigma_{01}=2.5 \mathrm{kPa}$. It is reasonably expected that the sample will liquefy with a couple of more cycles of loading. Although the total volume of the sample is maintained constant, there are local volume fluctuations at each Gauss point, which indicates that the deformation is not uniform at all over the problem domain. As seen from Figure 21(a), some locations in the sample experience dilation (void ratio larger than the initial value), whereas others undergo contraction (void ratio smaller than the initial value), although the difference is not significant (with the local void ratio ranging from 0.22 to 0.225 ). The contact network of the RVE at $\mathrm{N}^{\circ} 390$ at the final stage in Figure 21(b) indicates that the material point has already liquefied. The contacts are so few and weak and scattered that they cannot form any percolating force chains to sustain external shear.

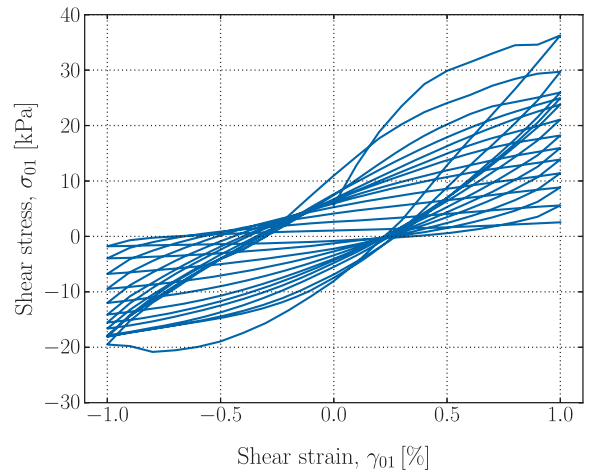

(a) Shear stress-strain relation

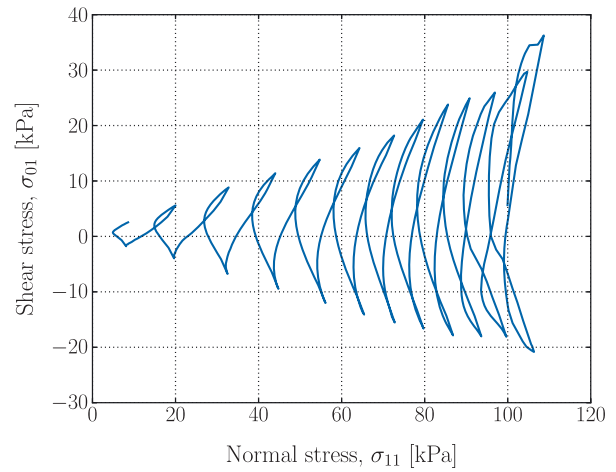

(b) Normal-shear stress relation

Figure 20. Global responses of the cyclic simple shear test controlled by a maximum shear strain $\gamma_{01}=1 \%$.

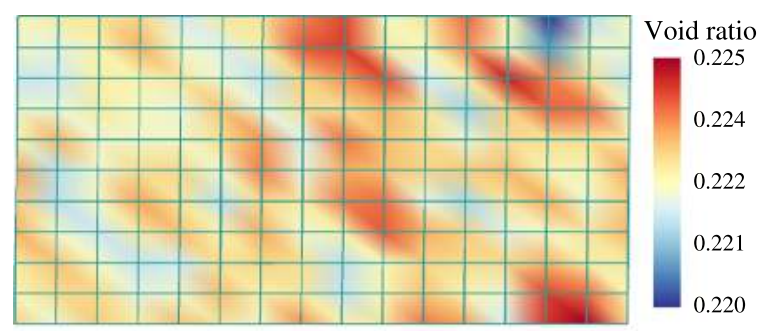

(a)

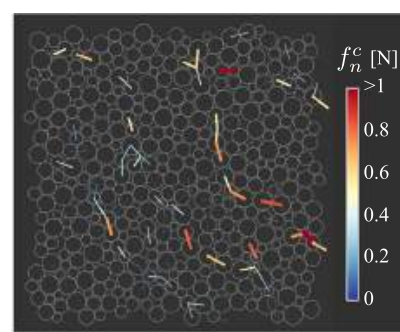

(b)

Figure 21. (a) Contour of void ratio at the final state after the maximum shear strain-controlled cyclic loading and (b) the structure and the force chains of $\mathrm{N}^{\circ} 390$. 


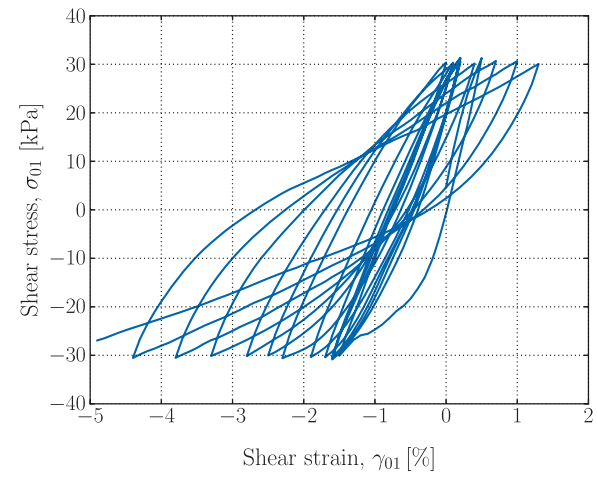

(a) Shear stress-strain relation

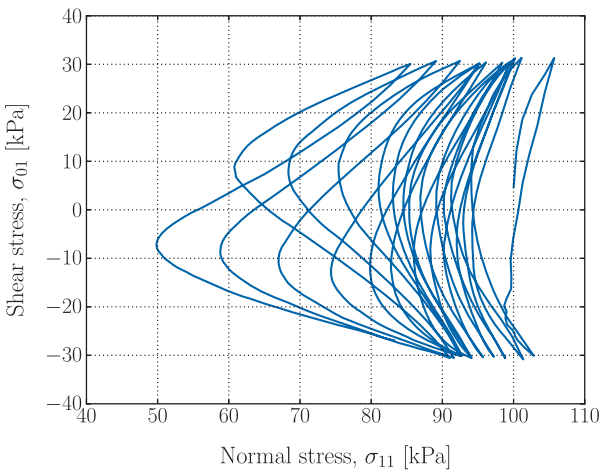

(b) Normal-shear stress relation

Figure 22. Global responses of the cyclic simple shear test under maximum shear stress control $\left|\sigma_{01}\right|=$ $30 \mathrm{kPa}$.

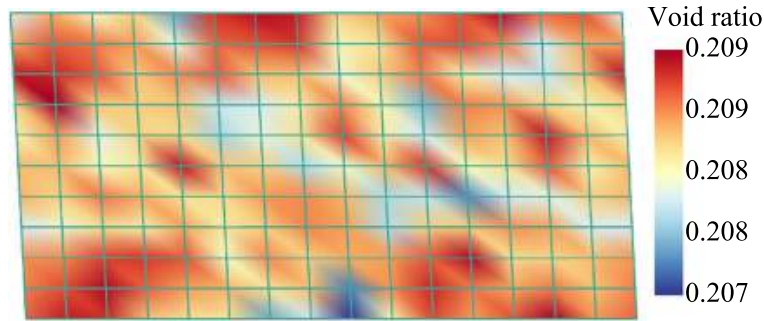

(a)

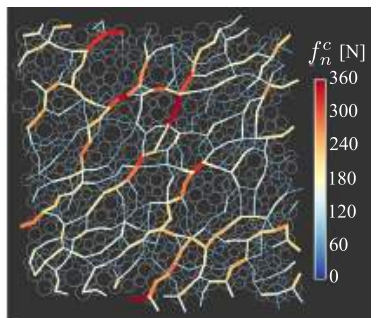

(b)

Figure 23. (a) Contour of void ratio at the final state after the maximum shear stress-controlled cyclic loading and (b) the structure and the force chains of $\mathrm{N}^{\circ} 390$.

\subsection{Maximum shear stress-controlled test}

The sample for this test is prepared to have an initial void ratio of 0.209 , which is slightly denser than the one in the previous strain-controlled test. The sample is then subject to stress-controlled cyclic shear. The loading is terminated when the accumulated shear strain $\left|\gamma_{01}\right|$ reaches 5\% after 13 cycles. The global stress-strain responses are shown in Figure 22, which are again consistent with experimental tests [64] and other model simulations [65] under the same loading conditions. Clearly, the overall response is not symmetric about the zero shear strain axis, and the sample is more contractive in the negative loading direction, which may be caused by fabric rearrangement of the material. Observed from the contour of void ratio in Figure 23(a), the deformation is relatively uniform in this test compared with the previous one. The void ratios slightly vary from 0.207 to 0.209 . At the final stage, the RVE at $\mathrm{N}^{\circ} 390$ shows several obvious strong force chains aligning along the diagonal direction of the packing. The pattern is reminiscent of that shown in a monotonic simple shear test. The maximum value of the contact normal force is more than two orders larger than that in the previous test ( $360 \mathrm{~N}$ against $\sim 1 \mathrm{~N}$ ). As the applied maximum shear stress is low, the sample will not totally lose its strength and strong force chains can be observed within the RVE packings. However, cyclic mobility takes place in this case as the accumulated shear strain becomes larger and larger. In practice, this phenomenon may also cause catastrophic hazards such as lateral spreading of slopes. According to some seismic design codes, this failure mode is also called liquefaction.

\section{CONCLUSIONS}

We have presented a hierarchical multiscale framework by coupling FEM and DEM for the simulation of cohesionless granular media. In the framework, the DEM is employed to capture the highly 
nonlinear and dissipative material response of typical granular media, whereas the continuum-based FEM is used to solve large-scale BVPs. A hierarchical solution scheme is developed for the coupling such that a DEM assembly is attached to each Gauss integration point of the FEM mesh to serve as a RVE, through which the DEM and FEM exchange information such as stress, strain and tangent modulus. Such a hierarchical structure enables us to successfully bypass the phenomenological manner in conventional constitutive modelling of granular media and meantime helps to retain the computational efficiency of FEM for solving BVPs. The efficiency of the hierarchical multiscale modelling approach can be greatly enhanced with the implementation of parallelisation for local DEM computing. Some key features of the proposed multiscale approach and novel findings from the study are summarised in the succeeding text.

(1) The solution procedure of the hierarchical multiscale approach involves a Newton-Raphson iteration scheme, wherein the tangent operator needed for the FEM solution can be estimated either from the elastic modulus of the DEM assembly or by using the perturbation method. Although both methods yield close global responses, the use of elastic modulus proves to be more stable and efficient in terms of convergence rate.

(2) Because the computation of the local DEM packing at each Gauss point is independent, the parallelisation of the proposed framework can be easily implemented, which is believed to be pivotal for the framework to be practically useful. A linear scalability is found for our parallelisation based on a high-performance computing cluster facility. Simulations of typical geotechnical BVPs prove the efficiency of the parallelised framework.

(3) Simulations of monotonic biaxial compression test and cyclic simple shear test on sand highlight the advantages of the proposed multiscale modelling approach. It is demonstrated that the modelling can adequately capture the typical responses of sand in these tests and meanwhile provides rich information at the micro-structural level to corroborate and explain the macroscopic behaviour of granular materials.

(4) Our multiscale simulations show that strain localisation can occur in a uniform sample with symmetric smooth boundary conditions without introducing any artificial imperfection. Inspection of the micro-structural data indicates that the mild anisotropy in the disordered RVE packing leads to non-coaxial response of the sample, which serves as a mechanism of symmetry breaker and triggers the initiation of strain localisation. Local analyses show that the local Gauss points outside the shear band may undergo unloading and develop relatively small deformations, whereas those inside the shear band may experience substantial shear deformation and reach the critical state. It is also found that a small proportion $(\sim 1.5 \%$ for the simulated test) of Gauss points fail along the edges of the shear band after very large deformation with unconfined dilation. The contact force networks for RVEs at Gauss points inside the shear band show more strong force chains penetrating the RVE domain which is aligned with the major compression direction, and there are virtually no contacts for the failed RVEs.

(5) Without resorting to sophisticated constitutive assumptions, the proposed multiscale framework is capable to model the hysteresis behaviour of granular media subject to cyclic loading. In constant volume tests controlled by the maximum shear strain, an initially loose sample goes to liquefaction after a number of shear cycles. Although the total volume of the sample is maintained constant, small local volume fluctuations caused by varied responses of RVEs in the FEM domain are observed. After liquefaction, the corresponding local RVE has only very few weak interparticle contacts. In the maximum shear stress-controlled cyclic shear test, the sample does not totally lose strength but undergoes cyclic mobility. These results are qualitatively consistent with observations from laboratory tests and other model simulations.

This study is focused on the formulation and calibration of the multiscale framework and demonstration of its predictive capability. Only brief discussion has been devoted to specific details of the approach, including the RVE calibration and mesh/element type dependencies. Nevertheless, we emphasise that when a particular BVP is treated, the RVE may need to be carefully calibrated. In addition to the method described in the paper, the specific size of RVE can also be determined by the convergence of RVE response to a steady one when the size is increased. To consider the effect of particle shape in real sand, more complex DEM models may be adopted (e.g. using particle clump 
and others). The FEM mesh size and element type need to be cautiously chosen, depending on the problem requirements. To eliminate or minimise the influence of mesh dependency, a possible solution would be to develop advanced formulations for granular media based on micropolar theory considering the couple stress and intrinsic length scale [12, 13, 66-68]. Furthermore, although the current framework has been presented in $2 \mathrm{D}$, it is readily applicable to a general 3D case if adequate computing resources are cheaply available (e.g. large number of cluster cores), because both Escript and YADE have been designed for 3D simulations and the pertaining formulations [i.e. Equations (5), (8) and (9)] are given in general 3D. All these endeavours are ongoing pursuits by the authors.

\section{APPENDIX : RELATING THE SMALL STRAIN FORMULATION WITH THE FINITE STRAIN FORMULATION}

The Piola-Kirchhoff stress $\boldsymbol{P}$ and the deformation gradient $\boldsymbol{F}=\partial \boldsymbol{x} / \partial \boldsymbol{X}$ are usually used in finite strain formulation in FEM, where $\boldsymbol{x}$ and $\boldsymbol{X}$ denote the current (deformed) and the reference (undeformed) geometries of the problem domain, respectively. And the global equilibrium equation to be solved is as follows

$$
\nabla_{\boldsymbol{X}} \cdot \boldsymbol{P}=\mathbf{0}
$$

As $\boldsymbol{P}$ can be linked with the Cauchy stress through $\boldsymbol{P}=J \boldsymbol{\sigma} \cdot \boldsymbol{F}^{-T}$ where $J=\operatorname{det} \boldsymbol{F}$ is the Jacobian, Equation (A.1) and the Cauchy equilibrium equation $\nabla \cdot \boldsymbol{\sigma}=\mathbf{0}$ are actually equivalent because

$$
\nabla_{\boldsymbol{X}} \cdot \boldsymbol{P}=J \nabla_{\boldsymbol{x}} \cdot \boldsymbol{\sigma}
$$

After the nodal displacements are obtained, the infinitesimal deformation $\nabla \boldsymbol{u}$ can be used to update the deformation gradient

$$
\boldsymbol{F}^{j}=(\boldsymbol{\delta}+\nabla \boldsymbol{u}) \boldsymbol{F}^{j-1}
$$

where the superscripts $j-1$ and $j$ denote the two consecutive loading steps. Then, instead of using the infinitesimal strain $\boldsymbol{\varepsilon}$, one can use the Green-Lagrangian strain $\boldsymbol{E}$ to measure the deformation level

$$
\boldsymbol{E}=\frac{1}{2}(\boldsymbol{C}-\boldsymbol{\delta})
$$

where $\boldsymbol{C}=\boldsymbol{F}^{T} \boldsymbol{F}$ is the right Cauchy-Green deformation tensor. It is seen that the current multiscale model can naturally accommodate large deformations (as long as the FEM mesh is not severely distorted) because no stress-strain integration is needed at the local material points and the boundary condition for local RVEs accounts for both strain and rotation.

\section{ACKNOWLEDGEMENTS}

The authors wish to thank Prof Jacques Desrues for sharing his recent paper to appear in Acta Geophysica which shows encouragingly consistent results with this study. We also benefit from the discussion with Prof Xikui Li on this topic during his visit to HKUST. The authors are grateful to Dr. Bruno Chareyre and Dr. Jan Stránský for the discussion and code contribution on parallelising YADE. They would also like to acknowledge the support from the Science School Computational Science Initiative, HKUST and the Research Grants Council of Hong Kong 623609. 


\section{REFERENCES}

1. Li X, Dafalias Y. Anisotropic critical state theory: the role of fabric. Journal of Engineering Mechanics 2012; 138(1):263-275.

2. Gao Z, Zhao J, Li XS, Dafalias YF. A critical state sand plasticity model accounting for fabric evolution. International Journal for Numerical and Analytical Methods in Geomechanics 2014; 38(4):370-390.

3. Gutierrez M, Ishihara K. Non-coaxiality and energy dissipation in granular material. Soils and Foundations 2000; 40:49-59.

4. Yu HS, Yuan X. On a class of non-coaxial plasticity models for granular soils. Proceedings of the Royal Society A 2006; 462(2067):725-748.

5. Tejchman J, Wu W. Non-coaxiality and stress-dilatancy rule in granular materials: FE investigation within micropolar hypoplasticity. International Journal for Numerical and Analytical Methods in Geomechanics 2009; 33: $117-142$.

6. Muhlhaus H, Moresi L, Gross L, Grotowski J. The influence of non-coaxiality on shear banding in viscous-plastic materials. Granular Matter 2010; 12:229-238.

7. Gao Z, Zhao J. Strain localization and fabric evolution in sand. International Journal of Solids and Structures 2013; 50:3634-3648.

8. Cundall PA. Strack ODL. A discrete numerical model for granular assemblies. Géotechnique 1979; 29(1):47-65.

9. Kouznetsova V, Geers MGD, Brekelmans WAM. Multi-scale constitutive modelling of heterogeneous materials with a gradient-enhanced computational homogenization scheme. International Journal for Numerical Methods in Engineering 2002; 54:1235-1260.

10. Miehe C, Dettmar J, Zäh D. Homogenization and two-scale simulations of granular materials for different microstructural constraints. International Journal for Numerical Methods in Engineering 2010; 83:1206-1236.

11. Geers MGD, Kouznetsova VG, Brekelmans WAM. Multi-scale computational homogenization: trends and challenges. Journal of Computational and Applied Mathematics 2010; 234:2175-2182.

12. Li X, Liu Q, Zhang J. A micro-macro homogenization approach for discrete particle assembly - Cosserat continuum modeling of granular materials. International Journal of Solids and Structures 2010; 47:291-303.

13. Li X, Zhang X, Zhang J. A generalized Hill's lemma and micromechanically based macroscopic constitutive model for heterogeneous granular materials. Computer Methods in Applied Mechanics and Engineering 2010; 199: 3137-3152.

14. Meier H, Steinmann P, Kuhl E. On the multiscale computation of confined granular media. In ECCOMAS Multidisciplinary Jubilee Symposium, Computational Methods in Applied Sciences, Vol. 14, Eberhardsteiner J, Hellmich C, Mang H, Périaux J (eds). Springer: Netherlands, 2009; 121-133.

15. Andrade JE, Avila CF, Hall SA, Lenoir N, Viggiani G. Multiscale modeling and characterization of granular matter: from grain kinematics to continuum mechanics. Journal of the Mechanics and Physics of Solids 2011; 59:237-250.

16. Nitka M, Combe G, Dascalu C, Desrues J. Two-scale modeling of granular materials: a DEM-FEM approach. Granular Matter 2011; 13:277-281.

17. Kouznetsova V, Brekelmans WAM, Baaijens FPT. An approach to micro-macro modeling of heterogeneous materials. Computational Mechanics 2001; 27:37-48.

18. Feyel F. A multilevel finite element method $\left(\mathrm{FE}^{2}\right)$ to describe the response of highly non-linear structures using generalized continua. Computer Methods in Applied Mechanics and Engineering 2003; 192:3233-3244.

19. Verhoosel CV, Remmers JJC, Gutiérrez MA, de Borst R. Computational homogenization for adhesive and cohesive failure in quasi-brittle solids. International Journal for Numerical Methods in Engineering 2010; 83:1155-1179.

20. Meier HA, Steinmann P, Kuhl E. Towards multiscale computation of confined granular media - contact forces, stresses and tangent operators. Technische Mechanik 2008; 28(1):32-42.

21. Andrade JE, Tu X. Multiscale framework for behavior prediction in granular media. Mechanics of Materials 2009; 41:652-669.

22. Chen Q, Andrade JE, Samaniego E. AES for multiscale localization modeling in granular media. Computer Methods in Applied Mechanics and Engineering 2011; 200:2473-2482.

23. Guo N, Zhao J. A hierarchical model for cross-scale simulation of granular media. AIP Conference Proceedings 2013; 1542:1222-1225.

24. Nguyen TK, Combe G, Caillerie D, Desrues J. Modeling of a cohesive granular materials by a multi-scale approach. AIP Conference Proceedings 2013; 1542:1194-1197.

25. Munjiza AA. The Combined Finite-Discrete Element Method. Wiley: Chichester, UK, 2004.

26. Munjiza AA, Knight EE, Rougier E. Computational Mechanics of Discontinua, Wiley Series in Computational Mechanics. Wiley: Chichester, UK, 2012.

27. Xiang J, Munjiza A, Latham JP. Finite strain, finite rotation quadratic tetrahedral element for the combined finitediscrete element method. International Journal for Numerical Methods in Engineering 2009; 79:946-978.

28. Munjiza A, Lei Z, Divic V, Peros B. Fracture and fragmentation of thin shells using the combined finite-discrete element method. International Journal for Numerical Methods in Engineering 2013; 95:478-498.

29. Xiao SP, Belytschko T. A bridging domain method for coupling continua with molecular dynamics. Computer Methods in Applied Mechanics and Engineering 2004; 193:1645-1669.

30. Aubertin P, Réthoré J, de Borst R. Energy conservation of atomistic/continuum coupling. International Journal for Numerical Methods in Engineering 2009; 78:1365-1386. 
31. Aubertin P, Réthoré J, de Borst R. A coupled molecular dynamics and extended finite element method for dynamic crack propagation. International Journal for Numerical Methods in Engineering 2010; 81:72-88.

32. Wellmann C, Wriggers P. A two-scale model of granular materials. Computer Methods in Applied Mechanics and Engineering 2012; 205-208:46-58.

33. Christoffersen J, Mehrabadi MM, Nemat-Nasser S. A micromechanical description of granular material behavior. Journal of Applied Mechanics 1981; 48:339-344.

34. Borja RI, Wren JR. Micromechanics of granular media Part I: generation of overall constitutive equation for assemblies of circular disks. Computer Methods in Applied Mechanics and Engineering 1995; 127:13-36.

35. Wren JR, Borja RI. Micromechanics of granular media Part II: overall tangential moduli and localization model for periodic assemblies of circular disks. Computer Methods in Applied Mechanics and Engineering 1997; 141: 221-246.

36. Kruyt N, Rothenburg L. Statistical theories for the elastic moduli of two-dimensional assemblies of granular materials. International Journal of Engineering Science 1998; 36:1127-1142.

37. Luding S. Micro-macro transition for anisotropic, frictional granular packings. International Journal of Solids and Structures 2004; 41:5821-5836.

38. Gross L, Bourgouin L, Hale AJ, Mühlhaus HB. Interface modeling in incompressible media using level sets in Escript. Physics of the Earth and Planetary Interiors 2007; 163:23-34.

39. Šmilauer V, Catalano E, Chareyre B, Dorofeenko S, Duriez J, Gladky A, Kozicki J, Modenese C, Scholtès L, Sibille L, Stránský J, Thoeni K. Yade Reference Documentation. In Yade Documentation, (1st edn), Šmilauer V (ed.)., 2010. The Yade Project.

40. Cundall PA, Hart R. Numerical modelling of discontinua. Engineering Computations 1992; 9:101-113.

41. O'Sullivan C, Bray JD. Selecting a suitable time step for discrcete element simulations that use the central difference time integration scheme. Engineering Computations 2004; 21:278-303.

42. Jop P, Forterre Y, Pouliquen O. A constitutive law for dense granular flows. Nature 2006; 441:727-730.

43. Miehe C, Dettmar J. A framework for micro-macro transitions in periodic particle aggregates of granular materials. Computer Methods in Applied Mechanics and Engineering 2004; 193:225-256.

44. Zhao J, Guo N. Rotational resistance and shear-induced anisotropy in granular media. Acta Mechanica Solida Sinica 2014; 27(1):1-14.

45. Mollon G, Zhao J. Fourier-Voronoi-based generation of realistic samples for discrete modeling of granular materials. Granular Matter 2012; 14:621-638.

46. Sloan SW. Substepping schemes for the numerical integration of elastoplastic stress-strain relations. International Journal for Numerical Methods in Engineering 1987; 24:893-911.

47. Zhao J, Sheng D, Rouainia M, Sloan SW. Explicit stress integration of complex soil models. International Journal for Numerical and Analytical Methods in Geomechanics 2005; 29:1209-1229.

48. Krabbenhoft K, Lyamin AV. Computational cam clay plasticity using second-order cone programming. Computer Methods in Applied Mechanics and Engineering 2012; 209-212:239-249.

49. Weatherley DK, Boros VE, Hancock WR, Abe S. Scaling benchmark of ESyS-Particle for elastic wave propagation simulations. IEEE Sixth International Conference on e-Science, Brisbane, Australia, 7-10 December 2010; $277-283$.

50. Visseq V, Alart P, Dureisseix D. High performance computing of discrete nonsmooth contact dynamics with domain decomposition. International Journal for Numerical Methods in Engineering 2013; 96:584-598.

51. Andrade JE, Borja RI. Capturing strain localization in dense sands with random density. International Journal for Numerical Methods in Engineering 2006; 67:1531-1564.

52. Andrade JE, Baker JW, Ellison KC. Random porosity fields and their influence on the stability of granular media. International Journal for Numerical and Analytical Methods in Geomechanics 2008; 32:1147-1172.

53. Tejchman J, Górski J. Finite element study of patterns of shear zones in granular bodies during plane strain compression. Acta Geotechnica 2010; 5:95-112.

54. Oda M, Kazama H. Microstructure of shear bands and its relation to the mechanisms of dilatancy and failure of dense granular soils. Géotechnique 1998; 48(4):465-481.

55. Satake M. Fabric tensor in granular materials. IUTAM Symposium on Deformation and Failure of Granular Materials, A.A, Balkema, Delft, 1982; 63-68.

56. Oda M. Fabric tensor for discontinuous geological materials. Soils and Foundations 1982; 22(4):96-108.

57. Roscoe KH, Schofield AN, Wroth CP. On the yielding of soils. Géotechnique 1958; 8(1):22-53.

58. Schofield AN, Wroth CP. Critical State Soil Mechanics. McGraw-Hill: London, UK, 1968.

59. Mooney MA, Finno RJ, Viggiani MG. A unique critical state for sand? Journal of Geotechnical and Geoenvironmental Engieering 1998; 124:1100-1108.

60. Desrues J, Chambon R, Mokni M, Mazerolle F. Void ratio evolution inside shear bands in triaxial sand specimens studied by computed tomography. Geotechnique 1996; 46(3):529-546.

61. Fu P, Dafalias YF. Study of anisotropic shear strength of granular materials using DEM simulation. International Journal for Numerical and Analytical Methods in Geomechanics 2011; 35:1098-1126.

62. Guo N, Zhao J. The signature of shear-induced anisotropy in granular media. Computers and Geotechnics 2013; 47:1-15.

63. Zhao J, Guo N. Unique critical state characteristics in granular media considering fabric anisotropy. Géotechnique 2013; 63(8):695-704.

64. Yang J. Sze HY. Cyclic behaviour and resistance of saturated sand under non-symmetrical loading conditions. Géotechnique 2011; 61(1):59-73. 
65. Borja RI, Lin CH, Montáns FJ. Cam-clay plasticity, Part IV: implicit integration of anisotropic bounding surface model with nonlinear hyperelasticity and ellipsoidal loading function. Computer Methods in Applied Mechanics and Engineering 2001; 190:3293-3323.

66. Ehlers W, Ramm E, Diebels S, D’Addetta GA. From particle ensembles to Cosserat continua: homogenization of contact forces towards stresses and couple stresses. International Journal of Solids and Structures 2003; 40: 6681-6702.

67. Kruyt NP. Statics and kinematics of discrete Cosserat-type granular materials. International Journal of Solids and Structures 2003; 40:511-534.

68. Li X, Wan K. A bridging scale method for granular materials with discrete particle assembly - Cosserat continuum modeling. Computers and Geotechnics 2011; 38:1052-1068. 